\title{
choix de la profondeur de reconnaissance pour les fondations superficielles
}

par

\author{
D. Cordary, J.-P. Giroud ${ }^{(*)}$, J.-P. Obin \\ Institut de Mécanique \\ Université de Grenoble
}

\section{Liste des notations}

\begin{tabular}{|c|c|}
\hline B & : largeur d'une fondation \\
\hline$B_{0}$ & $\begin{array}{l}\text { : largeur de référence servant à estimer les } \\
\text { effets du tassement différentiel (distance } \\
\text { entre semelles, côté du radier...) }\end{array}$ \\
\hline & : cohésion du sol \\
\hline$*$ & $\begin{array}{l}\text { : cohésion de la couche inférieure dans le } \\
\text { cas d'un sol bicouche }\end{array}$ \\
\hline & : profondeur de la base de la fondation \\
\hline oed & : module oedométrique \\
\hline$E_{1}$ (Eoed1) & $\begin{array}{l}\text { : module d'Young (Module oedométrique) } \\
\text { de la couche de sol situé sous la fondation }\end{array}$ \\
\hline${ }_{2}$ (Eoed2) & $\begin{array}{l}\text { module d'Young (module oedométrique) } \\
\text { de la couche de sol très compressible } \\
\text { située sous la couche de module } E_{1}\end{array}$ \\
\hline & : coefficient de sécurité \\
\hline 1 & $\begin{array}{l}\text { : épaisseur de la couche de sol située sous } \\
\text { la fondation }\end{array}$ \\
\hline$H_{1}$ & $\begin{array}{l}\text { hauteur de la zone concernée par la rup- } \\
\text { ture, sous la fondation, dans le cas d'un } \\
\text { sol homogène }\end{array}$ \\
\hline $\mathrm{H}_{2}$ & $\begin{array}{l}\text { épaisseur de la couche de sol très com- } \\
\text { pressible située sous la couche qui est en } \\
\text { contact avec la fondation }\end{array}$ \\
\hline$H_{r}$ & $\begin{array}{l}\text { : profondeur des sondages de reconnais- } \\
\text { sances, comptée à partir de la base de la } \\
\text { fondation }\end{array}$ \\
\hline & : longueur d'une fondation \\
\hline m & $\begin{array}{l}\text { : rapport entre l'augmentation de con- } \\
\text { trainte effective } \Delta \sigma^{\prime} \text { zz due au chargement } \\
\text { de la fondation et la contrainte effective } \\
\text { due au poids des terres } \sigma^{\prime} \text { zzo }\end{array}$ \\
\hline & $\begin{array}{l}\text { : coefficient de force portante de la formule } \\
\text { de Terzaghi }\end{array}$ \\
\hline
\end{tabular}
$\mathrm{O}_{2} \quad$ : coefficient sans dimension permettant le calcul des contraintes sous une fondation rectangulaire uniformément chargée reposant sur un sol'semi-infini.
$\mathrm{p}_{\mathrm{lim}} \quad$; pression portante du sol
$\mathrm{p}_{\lim 1} \quad$ : pression portante d'un sol homogène de caractéristiques $c$ et $\varphi$
$\mathrm{p}_{\text {lim2 }} \quad$ : pression portante d'un sol bicouche dont la première couche a pour caractéris- tiques $\mathrm{c}$ et $\varphi$
$\mathrm{p}_{\text {lim* }} \quad$ : pression portante d'un sol homogène de caractéristiques $c^{*}$ et $\varphi^{*}$
: pression admissible du sol
$\mathrm{p}_{\text {adm }}$
$P_{\text {adm } 1}$
$\mathrm{p}_{\text {adm2 }}$
p
; pression admissible d'un sol homogène de caractéristiques $c$ et $\varphi$
: pression admissible d'un sol bicouche : (la première couche a pour caractéristiques c et $\varphi$, la seconde $c^{*}=0, \varphi^{*}=0$ )
:pression moyenne à la base de la fondation

q

W

$\Delta w$

$\Delta w_{2}$

$\Delta w_{1}$

$\triangle \mathrm{w}_{\text {adm }}$

: surcharge du sol de fondation obtenue en déduisant de $\mathrm{p}$ la pression des terres enlevées

: tassement de la surface du sol

: accroissement du tassement que provoquerait l'existence d'une couche de sol très compressible

: contribution apportée au tassement de la surface par une couche de sol très compressible

; contribution apportée au tassement de la surface par une couche de même épaisseur que la couche définie à propos de $\Delta w_{2}$ mais de mêmes propriétés que le sol environnant

: accroissement admissible du tassement que provoquerait l'existence d'une couche de sol très compressible 
: profondeur, d'un point du sol, comptée à partir de la base de la fondation

$\gamma$

$\gamma$

$$
\gamma^{\prime}
$$

$$
\varphi
$$

: poids volumique du sol situé sous la fondation

: poids volumique déjaugé du sol situé sous la fondation

: poids volumique du sol situé entre la surface et le niveau de la base de la fondation

: angle de frottement du sol

: angle de frottement de la couche inférieure dans le cas d'un sol bicouche

: coefficient de Poisson du sol

: coefficient sans dimension permettant de déterminer la profondeur des sondages de reconnaissance pour le critère en tassement

: composante normale de la contrainte

: composante verticale de la contrainte agissant sur une facette horizontale

: composante verticale de la contrainte effective due au poids des terres agissant sur une facette horizontale

; augmentation de la composante verticale de la contrainte effective, due au chargement de la fondation, agissant sur une facette horizontale

: composante tangentielle de la contrainte

L'établissement d'un programme de reconnaissance, pour étudier un sol de fondation, est un problème à la fois technique et économique. II n'est pas question, bien sûr, de reconnaître la totalité du sol. II faut donc procéder par sondages, ce qui introduit une double incertitude:

. incertitude sur le sol situé entre les sondages:

- incertitude sur le sol situé à une profondeur plus grande que celle atteinte par les sondages.

Ces deux incertitudes sont de natures différentes:

- la première incertitude est liée à une interpolation entre les différents sondages; dans de nombreux cas il est possible de faire cette interpolation sans grand risque d'erreur et, si l'on estime qu'il y a un risque, il est aisé de décider, sur le chantier, de faire un sondage supplémentaire ; tout ceci est de la pratique courante et il est inutile d'en parler plus longuement ici :

- la seconde incertitude est liée à une extrapolation et, de ce fait, elle est beaucoup plus grande que la première: toute extrapolation implique un risque qu'il s'agit de minimiser: l'objet de cette étude est de proposer quelques règles dans ce but.

Bien entendu, il est inutile de disposer d'une règle pour choisir la profondeur des sondages lorsque le but de la reconnaissance est simplement de vérifier un détail géologique dont l'existence est prévisible. C'est le cas, par exemple, si l'on a une couche compressible (argile, silt...) reposant sur un substratum relativement indéformable (rocher, graviers compacts, marnes dures...). II suffit alors de pousser la reconnaissance jusqu'au substratum et d'y pénétrer de quelques mètres.
Mais le problème est plus ardu lorsqu'il s'agit de reconnaître un terrain a priori inconnu, ou encore un terrain dont on sait qu'il est très erratique. II faut alors disposer d'un critère de choix. Pour cela, nous plaçant dans le cas des fondations superficielles, nous analysons différents risques d'instabilité (Première Partie) et de tassement (Deuxième Partie). Cette analyse nous conduit à proposer des critères de choix de profondeur de reconnaissance en fonction des caractéristiques du bâtiment (géométrie et charge) et de celles du sol qui apparait au voisinage de la surface et qui est susceptible de servir d'assise à des fondations superficielles (radiers, semelles).

A la fin de cette étude, nous présentons des exemples d'applications et des recommandations à l'intention des praticiens.

\section{Critère se rapportant au risque d'instabilité}

Notre but, dans cette première partie, est de déterminer la profondeur de sol à reconnaître pour faire le calcul de pression portante avec le maximum de sécurité. II est donc logique de s'appuyer sur des résultats concernant la pression portante et la profondeur de sol intervenant dans sa détermination.

1.1 Rappels de résultats concernant la pression portante des fondations et la profondeur de la zone de sol intervenant dans son calcul.

La théorie de la plasticité fournit un moyen de déterminer la pression portante d'un sol sous une fondation donnée, moyennant les hypothèses suivantes:

. la loi de comportement du sol est du type rigideparfaitement plastique :

- le critère de rupture est celui de Coulomb $\left(\tau=c+\sigma_{n} \operatorname{tg} \varphi\right.$, lorsque $\left.\varphi \neq 0\right)$ ou celui de Tresca $(\tau=C$, lorsque $\varphi=0)$;

- l'état de déformation est plan.

Commençons par le cas d'un sol homogène. II est bien connu, grâce à de nombreuses expériences et à des observations sur le terrain, que la rupture du sol se produit dans une zone limitée par une surface que nous appellerons « surface de rupture (Fig. 1). La profondeur (mesurée à partir de la base de la fondation) atteinte par cette surface de rupture dépend de nombreux paramètres (angle de frottement, $\varphi$, cohésion, $c$, et poids volumique, du sol ; largeur, B, de la fondation et profondeur, D, de sa base) mais on peut en simplifier le calcul en supposant $=0$. On obtient alors une majorante de cette profondeur que nous appelons $H_{1}$ et qui ne dépend que de $\varphi$ (Tableau 1).

\begin{tabular}{c|c}
\hline$\varphi$ & $\frac{\mathrm{H}_{1}}{\mathrm{~B}}$ \\
\hline 0 & 0.71 \\
5 & 0.79 \\
10 & 0.89 \\
15 & 1.01 \\
20 & 1.16 \\
25 & 1.35 \\
30 & 1.59 \\
35 & 1.90 \\
40 & 2.35 \\
45 & 3.00 \\
50 & 4.03 \\
\hline
\end{tabular}

Tableau 1 Hauteur de la zone concernée par la rupture dans le cas d'un sol homogène d'angle de frottement $\varphi$. $\left(H_{1}\right.$ et $B$ sont définis sur la figure 1$)$ 


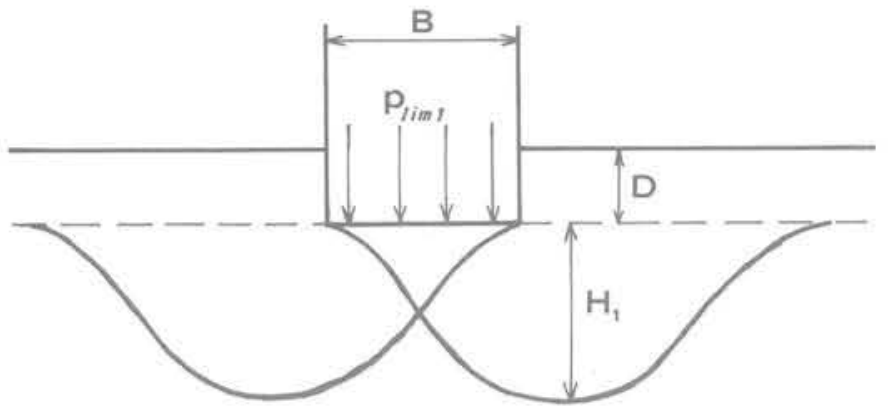

Fig. 1 Schéma de la rupture du sol sous une fondation superficielle (cas du sol homagène)

Ainsi dans le cas du sol homogène, nous connaissons la profondeur de sol intervenant dans le calcul de la pression portante. Ce cas est intéressant à titre de référence mais il n'est évidemment pas le plus défavorable. De nombreux travaux $[1,2,3,4,5,6]$ ont montré, en effet, qu'il y a des cas de sols composés de deux couches pour lesquels la pression portante est plus faible et la profondeur de sol intéressée plus grande que dans le cas du sol homogène.

Pour cela nous allons considérer trois cas de sol bicouche présentant les caractéristiques communes suivantes:

la couche supérieure a pour caractéristiques c et $\varphi$ :

- l'interface se situe à la profondeur $\mathrm{H}$ au-dessous de la base de la fondation;

- la couche inférieure a une épaisseur infinie.

Dans chacun de ces trois cas nous comparerons $\mathrm{p}_{\text {lim2 } 2}$ et $p_{\text {limt }}$ :

- $\mathrm{p}_{\text {lim2 }}$ étant la pression portante du sol bicouche considéré :

. $\mathrm{p}_{\text {lim1 } 1}$ étant la pression portante du sol homogène de caractéristiques $c$ et $\emptyset$.

Premier cas de sol bicouche: la couche inférieure peut être considérée comme rigide (relativement à la couche supérieure), avec un interface de la meilleure qualité possible (la contrainte tangentielle, $\tau$, n'est limitée que par la loi de Coulomb, $\tau \leqslant c+\sigma_{n} \operatorname{tg} \varphi$ ) (Fig. 2a). Deux situations peuvent se présenter (Fig. $2 \mathrm{~b}$ ) :

. si $\mathrm{H}$ est plus grand que $\mathrm{H}_{1}$ (Tableau 1), la couche rigide profonde n'a aucune influence sur la rupture $\left(p_{\text {lim2 }}=p_{\text {lim1 } 1}\right)$ et la surface de rupture se situe entièrement dans la couche supérieure :

. si $\mathrm{H}$ est plus petit que $\mathrm{H}_{1}$, la présence de la couche rigide joue un rôle favorable $\left(p_{\text {lim2 }}>p_{\text {lim } 1}\right)$ et la surface de rupture est tangente à l'interface $[7,8,9]$.

Ce premier cas de sol n'est donc pas défavorable par rapport au cas du sol homogène. Cependant nous allons voir que l'on peut le rendre défavorable par une simple modification de l'interface.

Deuxième cas de sol bicouche : cas identique au précédent, à ceci près que l'interface présente le risque maximal de glissement, la contrainte tangentielle y étant nulle $(\tau=0)$. Dans la pratique cela peut être da, par exemple, à la présence, à l'interface, d'une mince couche d'argile saturée de cohésion négligeable (Fig. 3a). Trois situations peuvent alors se présenter :

si $\mathrm{H}$ est supérieure à une certaine borne (courbe 1 de la figure $3 b$ ) la couche inférieure n'a aucune influence $\left(p_{\lim 2}=p_{\lim 1}\right)$;

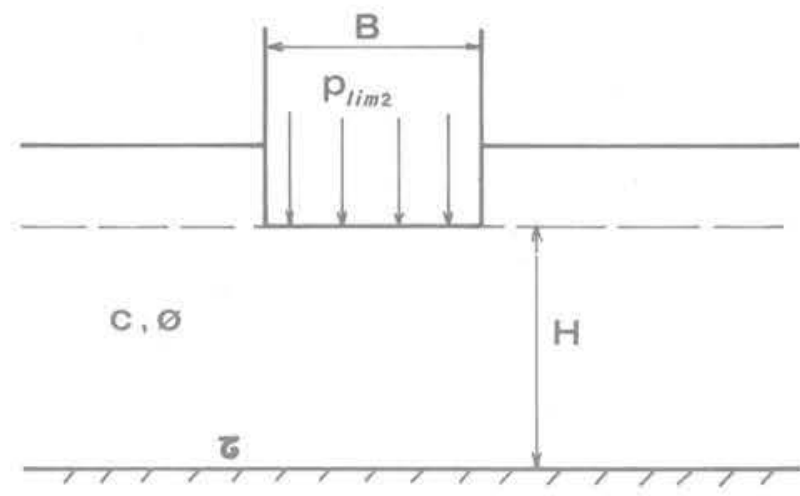

a

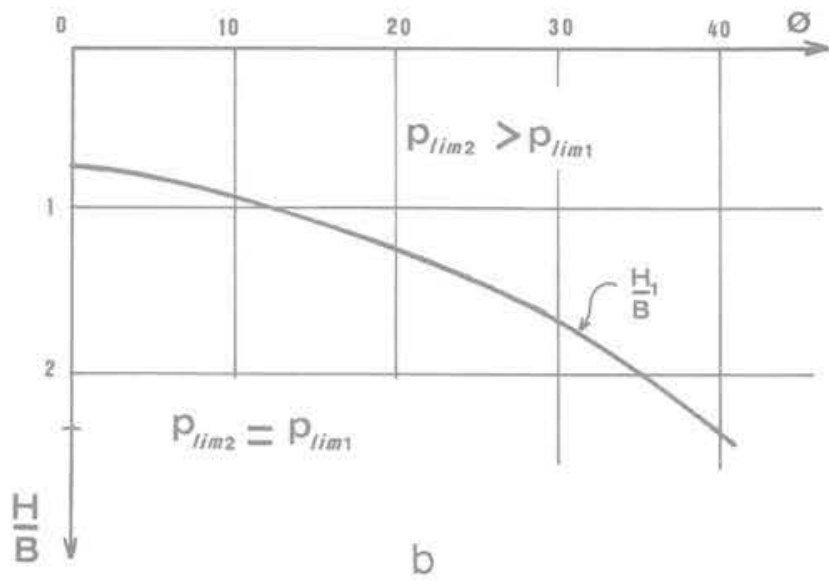

Fig. 2 (a) Sol homogène reposant sur un substratum rigide. Contact à l'interface : $\tau$ limité par la loi de Coulomb $\left(\tau \leqslant c+\sigma_{n} \operatorname{tg} \varphi\right)$

(b) Comparaison entre la pression portante $p_{\lim 2} d u$ cas ci-dessus et la pression portante $p_{\text {lim } 1}$ dans le cas du sol homogène infini (Nota : pour $\mathrm{H}_{1}$, voir la figure 1 et le tableau 1)

- si $\mathrm{H}$ est inférieure à la borne représentée par la courbe (2) de la figure $3 \mathrm{~b}$, la présence de la couche inférieure rigide (alors située très près de la fondation) améliore la force portante par rapport au cas du sol homogène $\left(p_{\text {lim2 }}>p_{\text {lim1 } 1}\right)$ :

- si $\mathrm{H}$ est compris entre ces deux bornes, la mauvaise adhérence à l'interface cause une diminution de la pression portante $\left(p_{\text {lim2 }}<p_{\text {lim } 1}\right)$.

En comparant la courbe (1) de la figure $3 \mathrm{~b}$ aux valeurs du Tableau 1 on constate que, dans le cas représenté par la figure $3 a$, la profondeur de sol intéressée risque d'être supérieure à celle relative au cas du sol homogène. (Nota : les courbes de la figure $3 \mathrm{~b}$ ne dépendent que de l'angle de frottement, $\varphi$, parce que le calcul a été fait par superposition des états d'équilibre limite $[8,9]$; en fait, ces courbes, relatives au terme $\mathrm{N}_{\mathrm{C}}$ de la formule de pression portante, donnent une majorante de $\mathrm{H}$ ). 


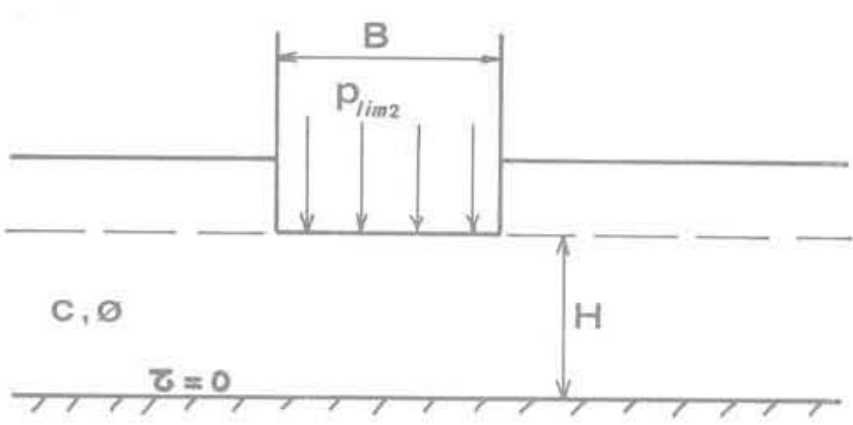

a

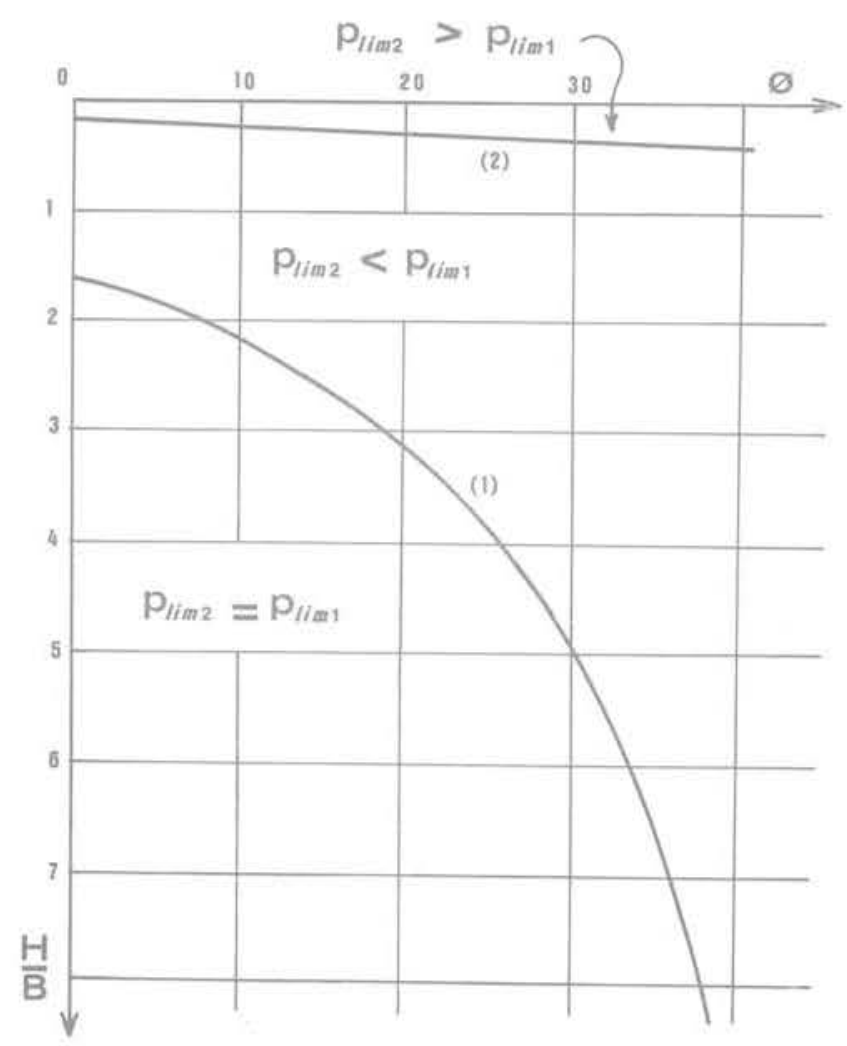

b

Fig. 3 (a) Sol homogène, reposant sur un substratum rigide. Contact à l'interface : $\tau=0$

(b) Comparaison entre la pression portante $p_{\text {lim } 2} d u$ cas ci-dessus et de la pression portante $p_{\lim 1}$ dans le cas du sol homogène infini.

Troisième cas de sol bicouche : les propriétés mécaniques $\left(c^{*}, \varphi^{*}\right)$ de la couche inférieure sont plus faibles que celles (c, $\varphi$ ) de la couche supérieure (Fig. 4a). Deux situations peuvent se présenter (Fig. 4b) :

. si $\mathrm{H}$ est supérieure à une certaine borne (qui dépend de la cohésion), la couche inférieure n'a pas d'influence $\left(p_{\lim 2}=p_{\lim 1}\right)$;

. si H est inférieure à cette borne, la présence de la couche inférieure modifie le schéma de rupture et la pression portante, $\mathrm{p}_{\mathrm{lim} 2}$, de ce sol bicouche se situe alors entre $\mathrm{p}_{\text {lim1 }}$ (calculée comme si le sol était homogène de caractéristiques $\mathrm{c}$ et $\varphi$ ) et $\mathrm{p}_{\mathrm{lim}}$ * (calculée comme si le sol était homogène de caractéristiques $c^{*}$ et $\varphi^{*}$ ).

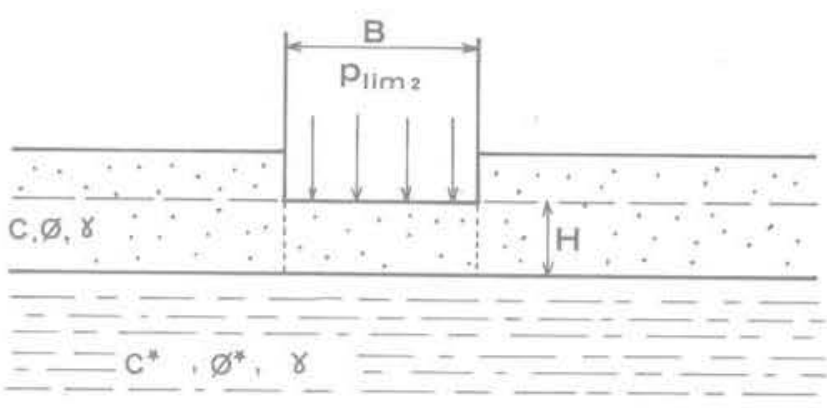

a

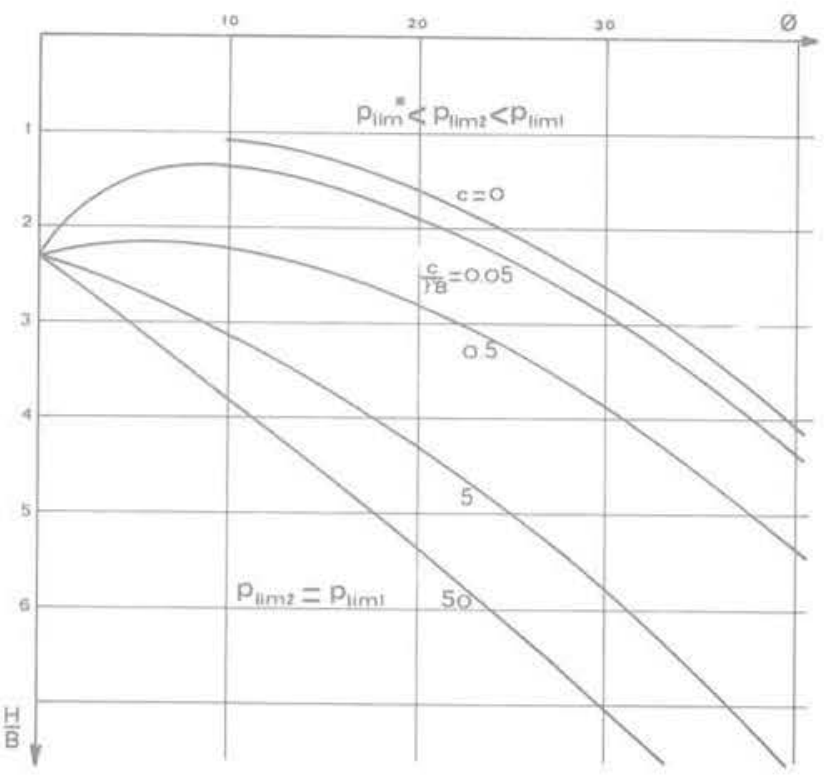

b

Fig. 4 (a) Sol de cohésion $c$, angle de frottement $\varphi$ et poids volumique $\gamma$, reposant sur un sol de cohésion $c^{*}$ et angle de frottement $\varphi^{*}$

(b) Comparaison entre la pression portante plim2 du sol ci-dessus et la pression portante du sol homogène infini

Les études expérimentales $[2,4]$ et théoriques $\mid 10,11$, $12,13 \mid$ montrent que $p_{1}$ dépend de paramètres géométriques $(H, B)$ et des propriétés du sol $\left(\gamma, \mathrm{C}, \varphi, \mathrm{c}^{*}, \varphi^{*}\right)$. On simplifie les calculs, et on se place dans le sens de la sécurité, en donnant à $c^{*}$ et $\varphi^{*}$ les valeurs les plus faibles possibles, c'est-à-dire $c^{*}=0$ et $\varphi^{*}=0$. On obtient ainsi la valeur maximale de la profondeur $\mathrm{H}$ à laquelle doit se trouver la couche de mauvaise qualité pour que la pression portante, $p_{\text {lim2 }}$, du sol bicouche soit plus faible que la

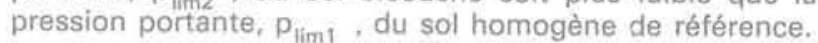

En conclusion, les deux derniers sols bicouches étudiés comportent un risque d'avair une force portante plus faible et une profondeur de sol intéressée plus grande que dans le cas de référence du sol homogène. Dans le cas 2, cela est dô à la mauvaise qualité de l'interface et, dans le cas 3 , 
à la mauvaise qualité de la couche inférieure. Les calculs concernant ces deux cas ont été faits de façons fort différentes $\left(^{*}\right)$, cependant on constate, en comparant la courbe (1) de la figure $3 b$ et les courbes de la figure $4 b$, que les ordres de grandeur sont les mêmes. Mais le cas 3 (Fig. 4b) a l'avantage de faire intervenir la cohésion: c'est donc celui-là seul que nous retiendrons dans la suite pour établir le critère.

1.2 Estimation de la profondeur de reconnaissance d'après la sécurité vis-à-vis de la pression portante.

D'après l'étude du paragraphe 1-1 on peut dire que la profondeur de sol à reconnaitre pour un projet de fondation dépend de l'existence éventuelle d'une couche sousjacente de mauvaise qualité. Mais le sol n'est pas seul en cause et la charge exercée par la fondation est également un paramètre essentiel du choix de la profondeur de reconnaissance. Soit $\mathrm{p}$ la pression moyenne prévue à la base de la fondation. La profondeur de reconnaissance doit être suffisante pour s'assurer que la pression admis sible, $\mathrm{p}_{\text {adm }}$, sur le sol est au moins égale à $\mathrm{p}$. Donc la reconnaissance minimale (celle que l'on cherche à définir) est telle que :

$$
p_{\text {adm }}=p
$$

L'ingénieur qui doit décider de la profondeur de reconnaissances à effectuer pour un projet de fondations ne connait, a priori, que le sol qui apparaît en surface et pour lequel il peut estimer les valeurs de $c$ et $\varphi$. II peut alors calculer la pression portante, $p_{\lim 1}$, quaurait le sol s'il était homogène de caractéristiques $c$ et $\varphi$. De cette pression portante, il déduit la pression admissible, $p_{\text {adm1 }}$, par la formule classique :

$$
p_{\text {adm } 1}-\gamma_{\circ D}=\frac{p_{\text {lim1 } 1}-\gamma_{0} D}{F}
$$

avec :

D : profondeur de la base de la fondation:

$\gamma_{0}$ : poids volumique du sol dans la couche d'épaisseur $D$ située entre la surface du sol et le niveau de la base de la fondation :

$\mathrm{F}$ : coefficient de sécurité, généralement pris égal à 3 .

Partant de là, trois cas peuvent se présenter:

. si la pression admissible ainsi calculée est inférieure à la pression requise, $\left(\mathrm{p}_{\text {adm1 } 1}<\mathrm{p}\right)$ il est impossible d'envisager de faire une fondation superficielle et il faut conduire la reconnaissance en vue de fondations profondes, ce qui sort du cadre de cette étude :

. si la pression admissible ainsi calculée est juste égale à la pression requise $\left(\mathrm{p}_{\mathrm{adm} 1} .=\mathrm{p}\right)$, la condition exprimée par la relation (1) est vérifiée et il faut faire la reconnaissance jusqu'à la profondeur indiquée par la figure $4 b$ pour s'assurer qu'il n'y a pas de sol de mauvaise qualité à une profondeur où il pourrait diminuer la pression portante, donc la pression admissible

. si la pression admissible ainsi calculée est supérieure à la pression requise $\left(\mathrm{p}_{\text {adm1 }}>\mathrm{p}\right)$, on peut tolérer qu'une couche de sol de mauvaise qualité diminue la pression portante donc la pression admissible.

(") Dans le cas 2, la théorie de la plasticité a été utilisée globalement dans la couche supérieure alors que, dans le cas 3 , la loi de plasticité de Cou lomb ou Tresca est écrite le long de la surface de rupture, supposée formée d'arcs circulaires, d'arc de spirale ou de plans. Nous avons montré que, dans le cas d'un milieu homogène, ces deux méthodes sont proches aussi bien en ce qui concerne la pression portante que la profondeur de sol intéressée $\mid 13,52-7$ et $2-8 \mid$. La cohésion c intervient dans les résultats relatifs au cas 3 (Fig. 4 b) car, contrairement au cas 2 , nous n'avons pas procédé par superposition.
Ce dernier cas étant le plus général, examinons-le en détail. La condition quí régit le choix de la profondeur de reconnaissance s'écrit alors :

$$
\mathrm{p}=\mathrm{p}_{\mathrm{adm2}}<\mathrm{p}_{\mathrm{adm} 1}
$$

Autrement dit la profondeur de reconnaissance doit être égale à l'épaisseur de la couche de sol de caractéristiques $c$ et $\varphi$, reposant sur un sol de caractéristiques $c=0$ et $\varphi=0$, l'ensemble ayant une pression admissible égale à $p$. Les valeurs numériques de cette profondeur de reconnaissance, $H_{r}$, sont données dans les figures 5 à 11 , en fonction des propriétés du sol et de la géométrie de la fondation. Ces résultats ont été établis à partir de nos travaux sur la pression portante des sols bicouches $|7,13|$. Quelques commentaires à propos de ces résultats:

- le coefficient de sécurité $\mathrm{F}$ a été pris égal à trois :

- la pression requise sous la fondation, $\mathrm{p}$, intervient en fait sous la forme $p-\gamma_{0} D$ car, comme dans la formule (2), on est certain que, quelle que soit la qualité du sol sous la fondation, la pression admissible vaudra au moins $\gamma_{0} \mathrm{D}$

- la courbe en tirets qui, sur les figures 5 à 8 , limite les courbes en traits pleins correspond au cas où la pression requise est juste égale à celle que peut fournir le sol superficiel (ces courbes en tirets sont donc identiques aux courbes de la figure $4 \mathrm{~b}$ ) :

, on constate que meilleur est le sol superficiel (plus $c$ et $\varphi$ sont grands), moins la reconnaissance devra être profonde car plus on pourra tolérer la présence d'une couche de mauvaise qualité.

Enfin, deux cas particuliers sont à signaler :

- lorsque le sol superficiel est sans frottement $(\varphi=0)$ la pression portante ne dépend pas de $\mathrm{c} / \gamma \mathrm{B}$ $\mid 7$ § $7-5,12,13$ \& $2-5 \mid$ et il est plus commode de présenter les variations de $\mathrm{H}_{r}$ en fonction de $\left(p-\gamma_{0} D\right) / c$ (Figure 9$)$; on voit que la courbe est presque une droite d'équation:

$$
\frac{H_{r}}{B}=1.25 \frac{\left(P-\gamma_{0} D\right)}{C}
$$

- lorsque le sol superficiel est sans cohésion, il est possible de rassembler dans la figure 10 les résultats disséminés dans les figures 5 à 8 ; la présentation s'en trouve simplifiée car $\mathrm{Hr} / \mathrm{B}$ ne dépend plus que de $\varphi$ et de $\left(p-\gamma_{0} D\right) / \gamma B$; pour des valeurs de $\varphi$ voisines de $40^{\circ}$ on a la formule approchée suivante :

$$
\frac{H_{r}}{B}=1.25 \sqrt{\frac{p-\gamma \circ D}{\gamma^{B}}}
$$

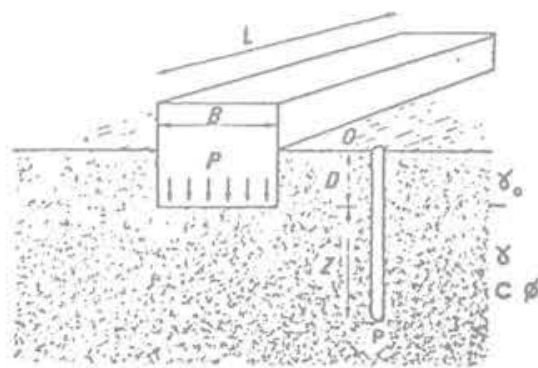

Fig. 5 Définition des termes utilisés dans les figures 6 à 11. Hr: profondeur de reconnaissance mesurée à partir de la base de la fondation; $c, \varphi, \gamma$ : cohésion, angle de frottement et poids volumique du sol situé immédia. tement sous la base de la fondation : $\gamma_{0}$ : poids volumique du sol situé entre la surface du sol et le niveau de la base de la fondation; $p$ : pression moyenne exercée par la fondation sur le sol (Nota : la longueur, L, n'intervient pas dans les calculs de pression portante mais elle interviendra dans la deuxième partie) 


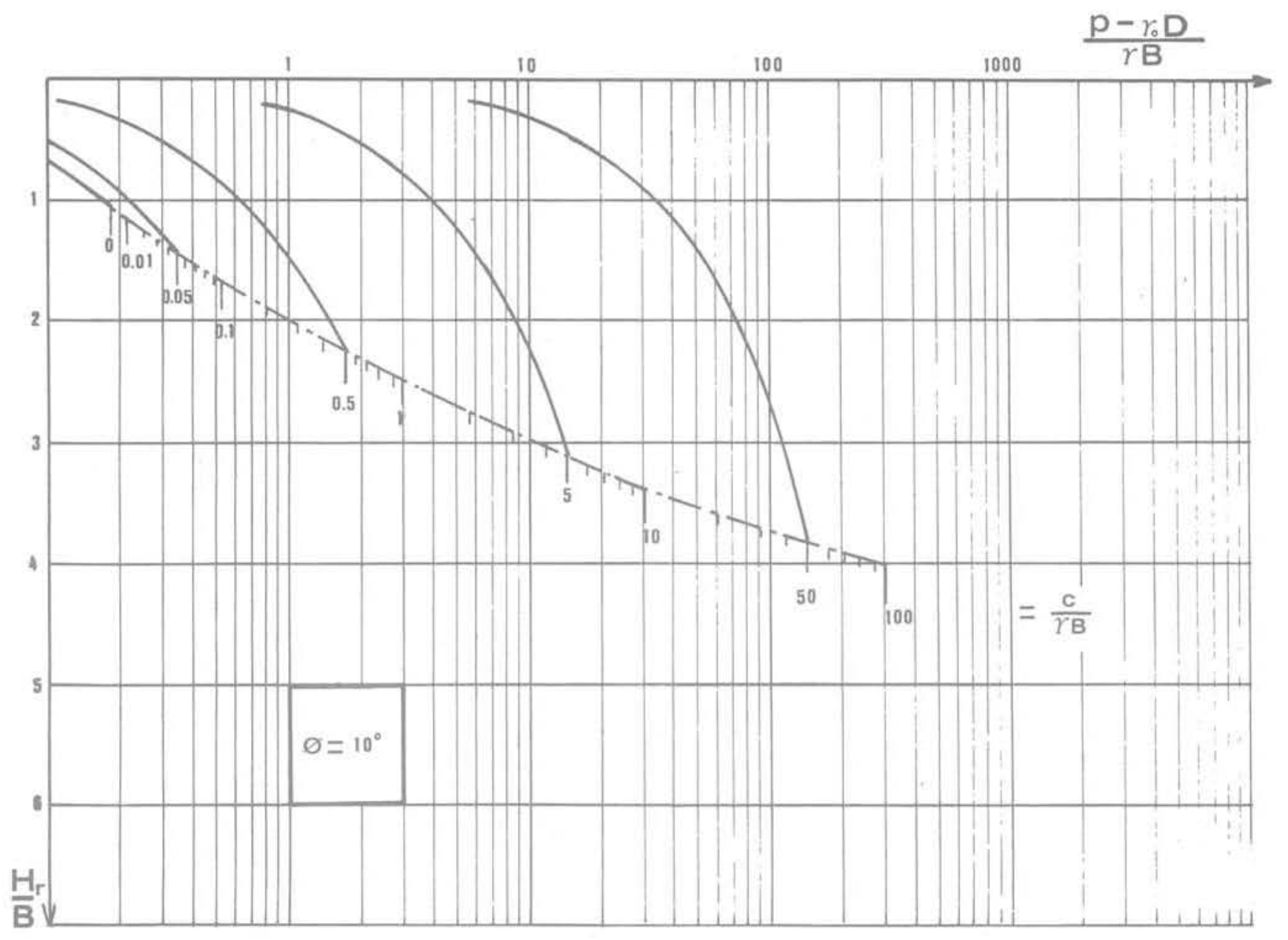

Fig. 6 et 7 Valeur de la profondeur de reconnaissance dans les cas $\varphi=10^{\circ}$ et $\varphi=20^{\circ}$. Voir la figure 5 pour la définition des termes

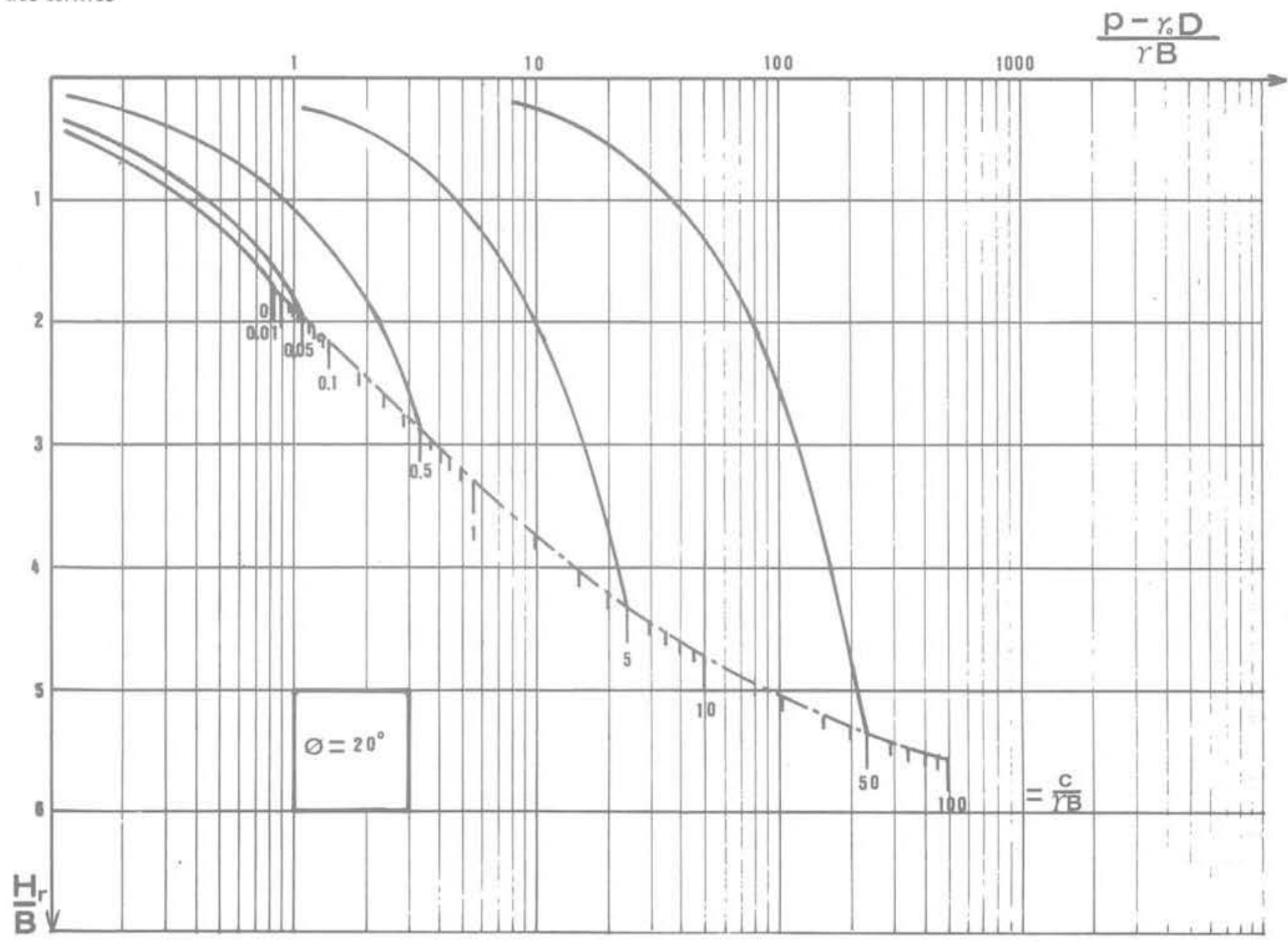




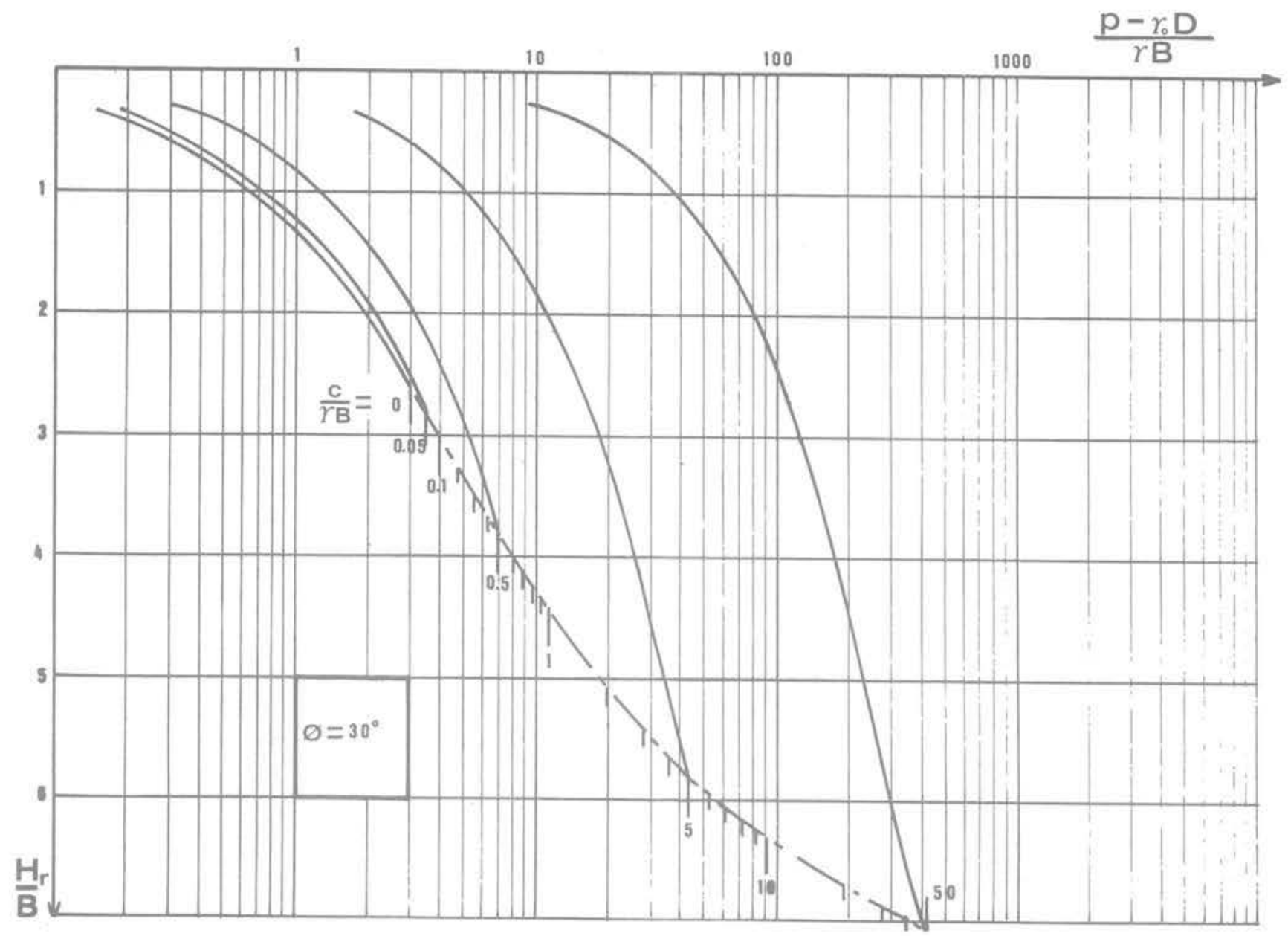

Fig. 8 et 9 Valeur de la profondeur de reconnaissance dans les cas $\varphi=30^{\circ}$ et $\varphi=40^{\circ}$. Voir la figure 5 pour la définition des termes

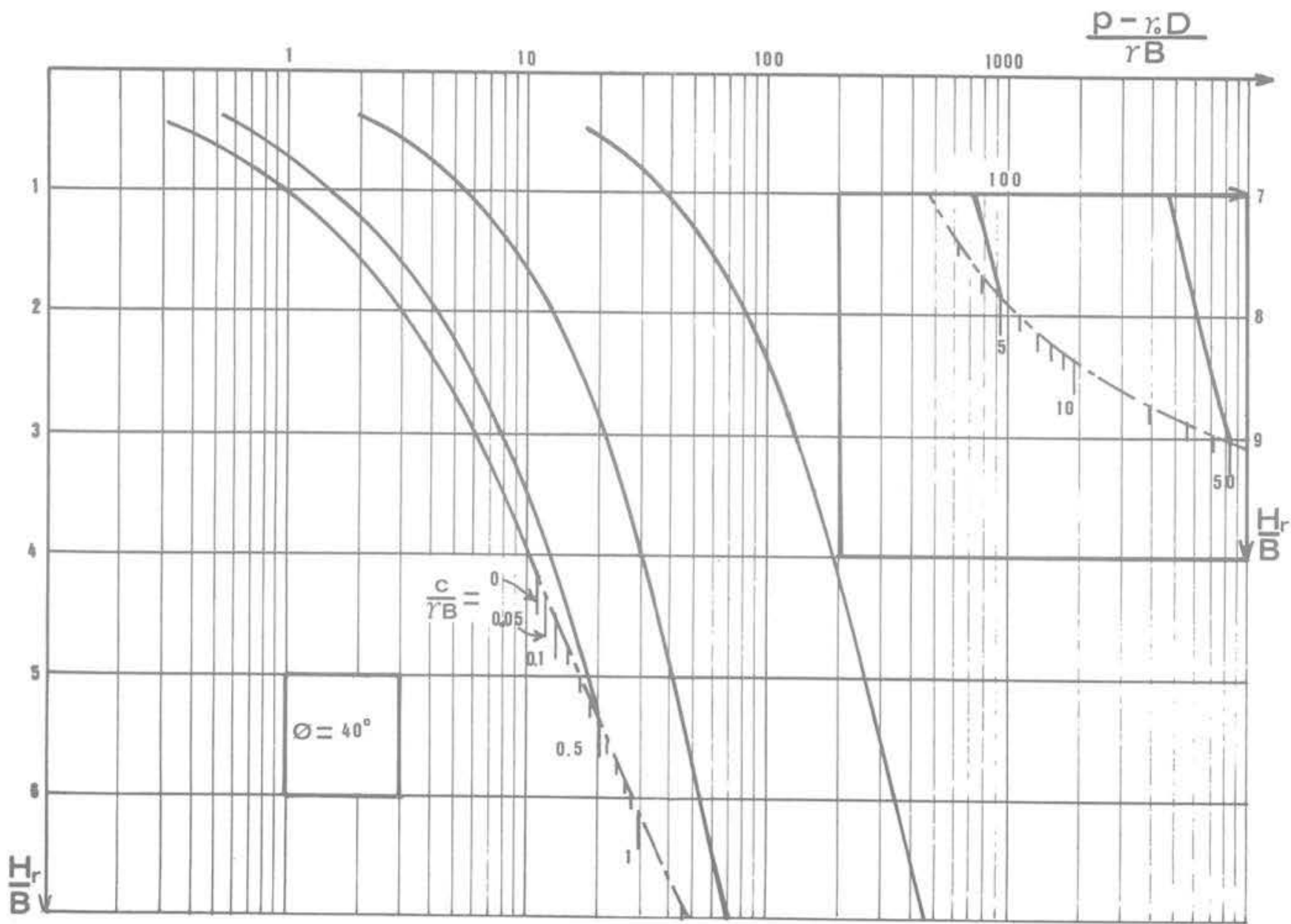



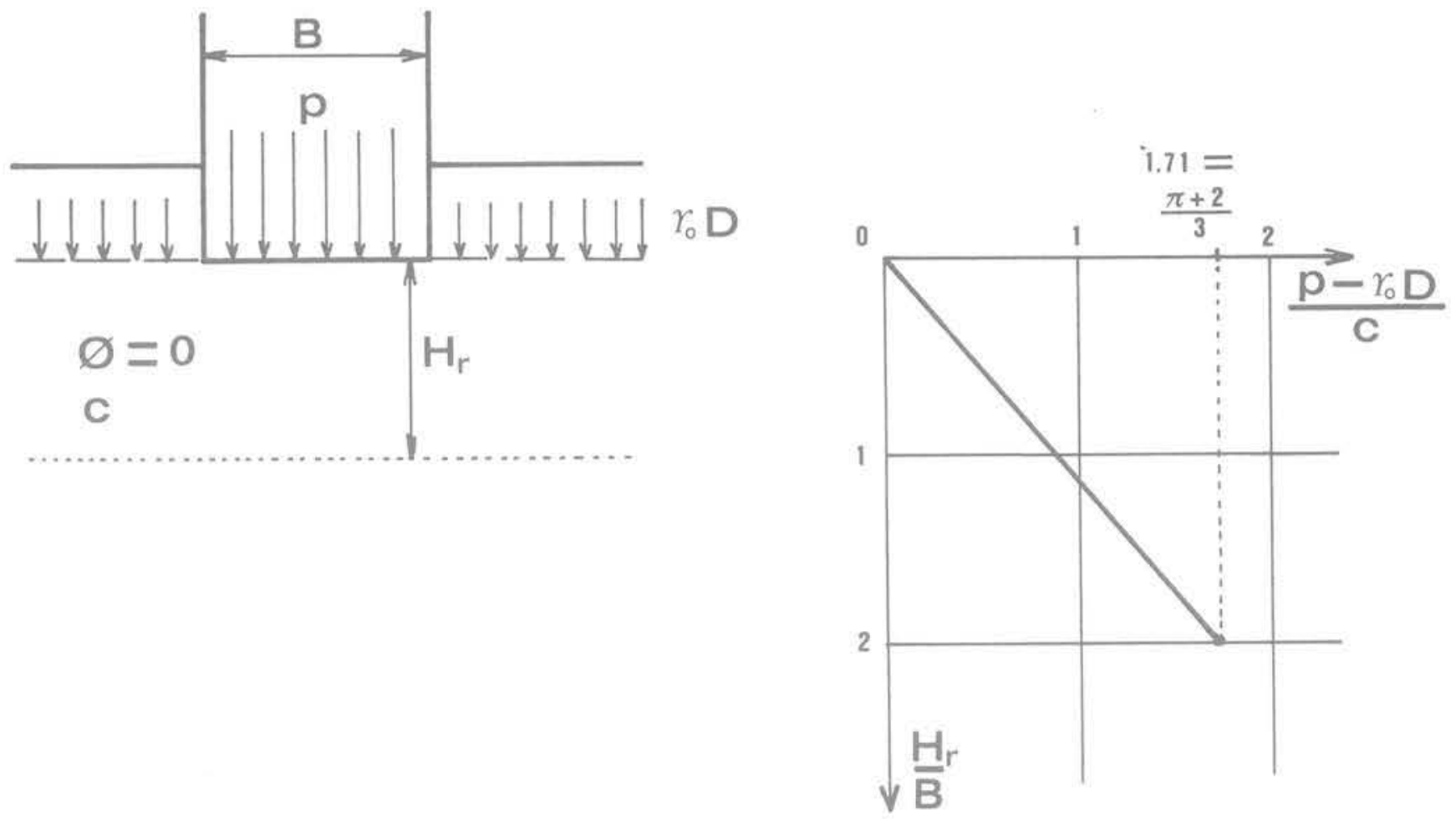

Fig. 10 Valeur de la profondeur de reconnaissance dans le cas $\varphi=0^{\circ}$. Voir la figure 5 pour la définition des termes

Fig. 11 Valeur de la profondeur de reconnaissance dans le cas $c=0$. Voir la figure 5 pour la définition des termes

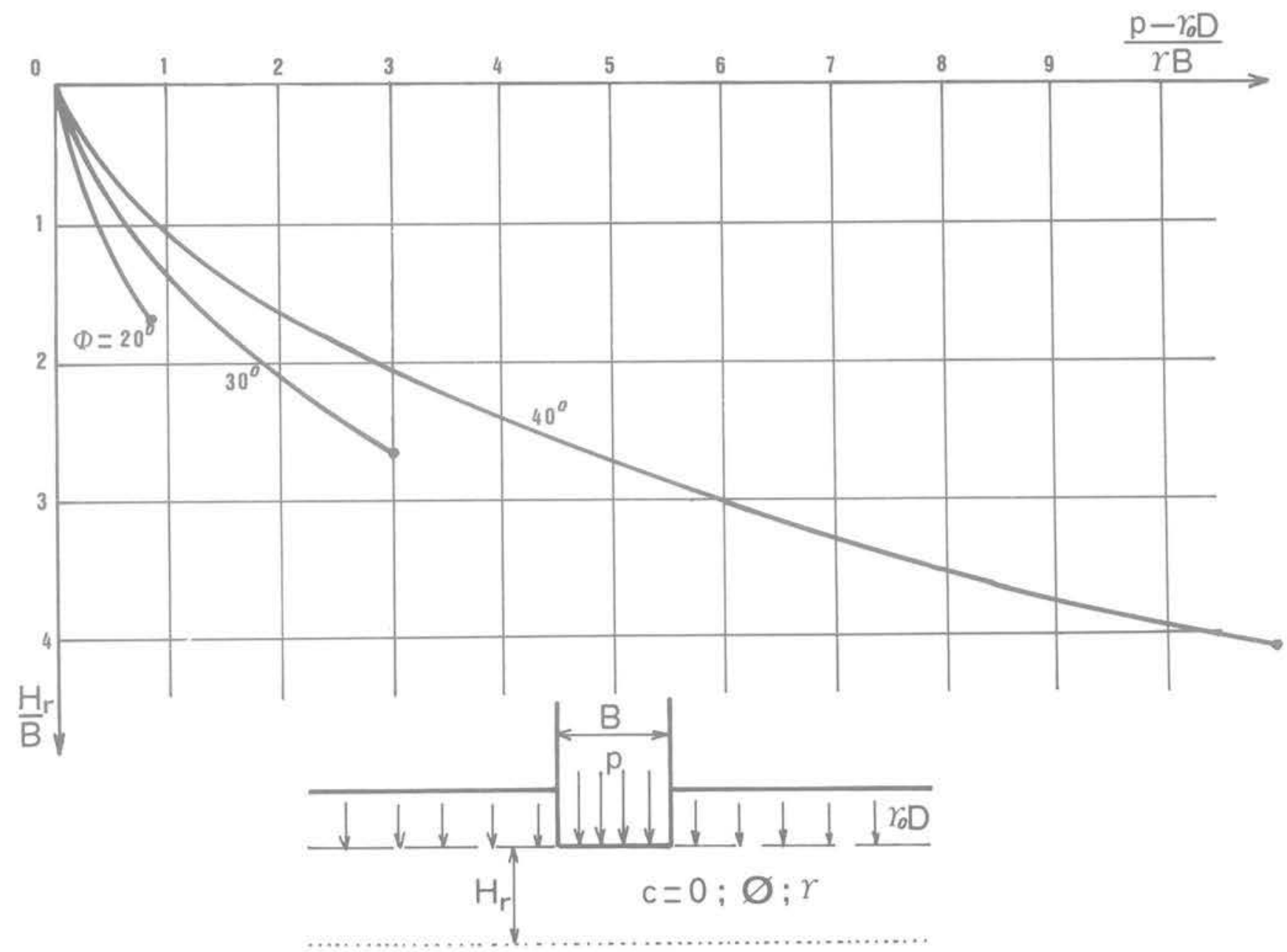




\section{Critère se rapportant au risque de tassement}

Nous venons de voir, dans la première partie, qu'il était possible de prendre en compte un " risque maximal " par rapport à la stabilité d'une fondation en supposant l'existence, à une certaine profondeur, d'une couche de sol de résistance nulle (c $=0, \varphi=0)$. II est tentant de procéder de même ici. Mais, en fait, le problème est différent : le défaut de stabilité s'exprime de manière absolue alors que les restrictions que l'on impose sur le tassement n'ont pas un caractère aussi brutal. Par ailleurs, le phénomène tassement fait intervenir un volume de sol beaucoup plus vaste que le phénomène rupture.

Essayons, malgré tout, d'appliquer au risque de tassement, correspondant à l'ignorance des propriétés du sol situé en dessous du niveau des sondages, une démarche voisine de celle de la première partie. Supposons en effet que l'on ait reconnu le sol jusqu'à la profondeur $\mathrm{H}$. Ce qui pourrait arriver de pire, c'est que le sol situé à une profondeur supérieure à $\mathrm{H}$ soit infiniment déformable quelle que soit la contrainte appliquée. Cette hypothèse est évidemment inacceptable car elle conduit à un tassement infini de la fondation quel que soit $\mathrm{H}$. Par ailleurs, elle est physiquement peu raisonnable puisque les propriétés mécaniques du sol finissent toujours par augmenter avec la profondeur. Dans ces conditions on est conduit obligatoirement à un certain arbitraire dans le choix du critère.

Comme les méthodes de calcul du tassement se divisent en deux catégories,

- les méthodes directes qui donnent directement le tassement.

- les méthodes indirectes qui permettent d'obtenir le tassement à partir des contraintes,

nous proposons, ici, deux critères complémentaires, l'un en contraintes, l'autre en tassement.

\subsection{Critère en contraintes}

Comme nous venons de le dire, les propriétés mécaniques des sols finissent toujours par s'améliorer avec la profondeur. Par conséquent la contribution d'une couche de sol au tassement d'une fondation a au moins deux raisons de diminuer quand la profondeur de cette couche augmente. En effet :

- l'augmentation de contrainte due à la fondation (qui est la cause de la déformation) se dissipe avec la profondeur :

- les propriétés mécaniques, et en particulier le module de déformation, augmentent avec la profondeur.

Malheureusement, ces constatations riont qu'un caractère qualitatif. Pour aboutir à des résultats quantitatifs il faut lier la variation des propriétés mécaniques à la profondeur.

Dans le cas d'un sol relativement homogène on peut remarquer que le module de déformation est d'autant plus grand que la contrainte de consolidation est plus élevée. Pour un sol normalement consolidé, celle-ci est égale à la contrainte effective due au poids des terres sus-jacentes. D'où l'idée, développée par Burmister $|14|$, que le risque tassement est d'autant plus faible que l'augmentation de la contrainte effective $\Delta \sigma_{z}^{\prime}$ due à la surcharge apportée par la fondation est petite par rapport à la contrainte verticale effective $\sigma_{z ́}$ due au poids des terres. On remarquera que ce critère tient compte simultanément des deux raisons invoquées plus haut.

Pratiquement cette condition peut s'exprimer de la manière suivante. On peut négliger le tassement dû aux couches situées au-dessous de la profondeur $\mathrm{H}$ à laquelle est vérifiée la relation (Fig. 12):

$$
\Delta \sigma_{z}^{\prime} \leqslant m \sigma_{z o}^{\prime}
$$

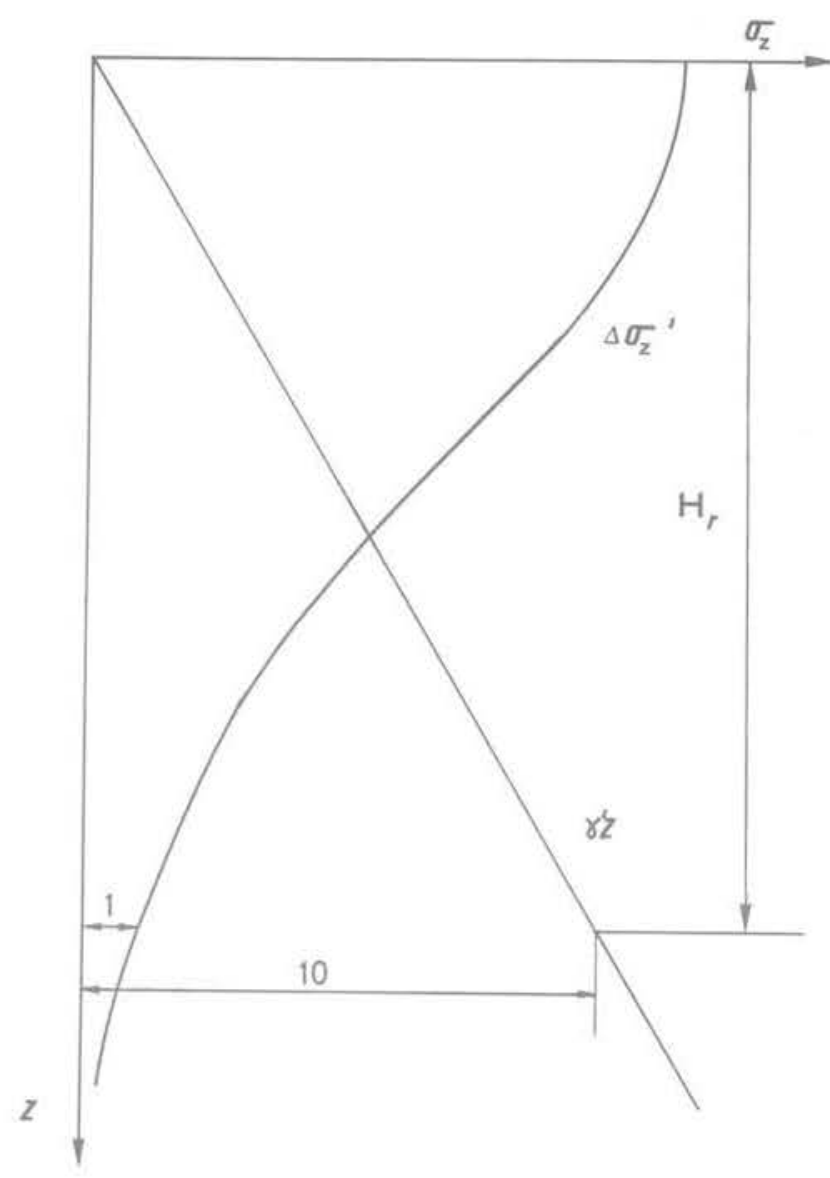

Fig. 12 Distribution dans le sol, en fonction de la profondeur $z$, de la contrainte effective $\sigma_{z o}^{\prime}=\gamma^{\prime} z$ due au poids des terres et de la contrainte effective $\Delta \sigma_{z}^{\prime}$ due à la surcharge apportée par la fondation. A la profondeur $H$, le rapport entre ces deux contraintes est $m$, d'après (6). $/ \mathrm{ci}, m=1 / 10$

avec :

$\Delta \sigma_{z}^{\prime}$ : contrainte verticale effective à la profondeur $\mathrm{H}$ due à la surcharge apportée par la fondation :

$\sigma_{z o}^{\prime}$ : contrainte verticale effective à la profondeur $\mathrm{H}$ due au poids des terres

$\mathrm{m}$ : coefficient dépendant du risque accepté.

Par exemple, Burmister recommande :

(7) $\quad m=0.1$ pour les sols fins (silts, argiles)

$\mathrm{m}=0.2$ pour les sols grossiers (sables, graviers)

Bien entendu, d'autres valeurs peuvent être envisagées le cas échéant.

On peut donc logiquement considérer que la valeur ains déterminée est une expression raisonnable de la profondeur des sondages de reconnaissance $\left(^{*}\right)$. Encore faut-il en donner une détermination pratique.

Pour un sol homogène sur une profondeur infinie l'augmentation de contrainte verticale due à une surcharge ne dépend pas des propriétés mécaniques (du moins si l'on fait l'hypothèse d'un comportement élastique et linéaire du sol). On peut alors représenter graphiquement la condition ci-dessus (Fig. 13) $[16,17]$.

(*) Cette condition est également utilisée dans les normes de calcul des fondations en usage en URSS $|18|$ pour limiter conventionnellement l'épaisseur de la couche compressible dans les méthodes de calcul du tassement. 


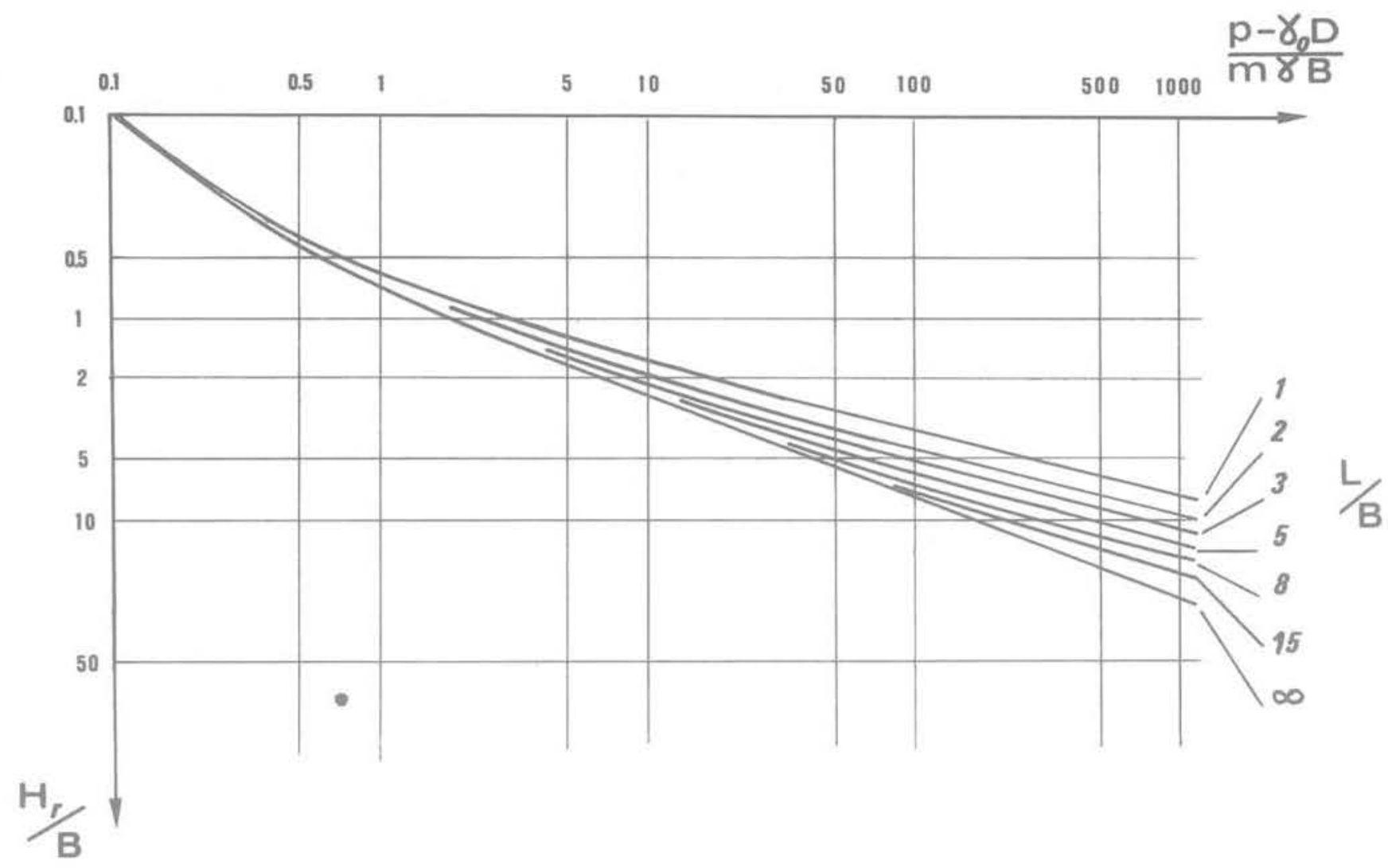

Fig. 13 Détermination de la profondeur de reconnaissance d'après le "critère en contraintes». Légende : L, longueur de la fondation; $m$ : coefficient lié au risque accepté (voir la relation 7). Pour la définition des autres termes, voir la figure 5

Fig. 14 Tassement au coin d'une fondation rectangulaire. Comparaison de la valeur w dans le cas d'un sol homogène et de la valeur $w+\Delta w$ dans le cas où une couche mince se trouve à la profondeur $H$
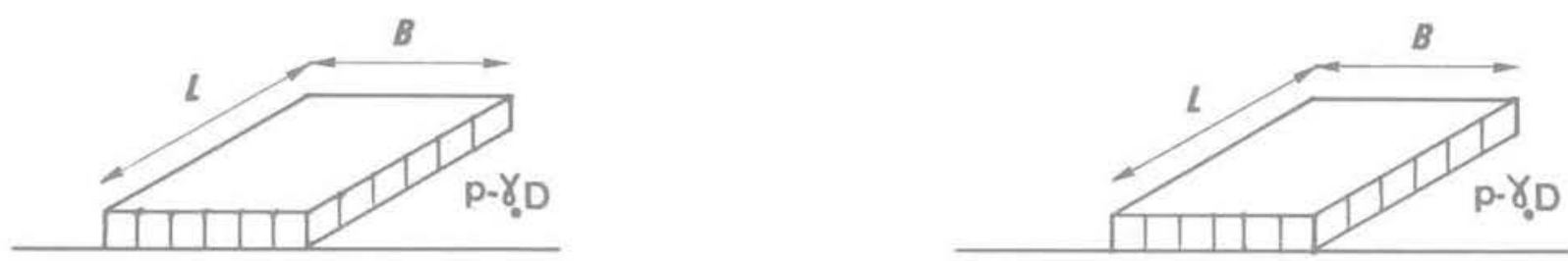

$E_{1}$

$E_{1}$

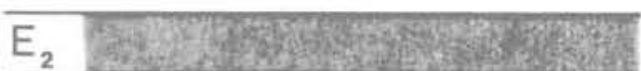

$\mathrm{H}_{2}$ 
En réalité le sol ne peut pas être homogène puisque l'on suppose implicitement en vérifiant la condition ci-dessus que ses propriétés s'améliorent avec la profondeur. Toutefois la distribution des contraintes verticales s'éloigne assez peu de celle du milieu homogène sauf dans le cas d'une couche dure reposant sur une couche molle épaisse. C'est justement un cas qui ne peut pas être traité avec ce critère car les propriétés mécaniques ne s'améliorent pas avec la profondeur. Dans la réalité il n'y a pas d'hésitation possible car s'il peut exister une couche compressible importante, les renseignements fournis par la géologie ne permettent pas de l'ignorer.

Hormis ce cas particulier qui ne peut pas passer inaperçu, et une autre configuration de sol plus insidieuse que nous étudierons dans le paragraphe suivant, ce critère est raisonnable car il est très stable: en effet si l'on rencontre une couche un peu plus compressible relativement épaisse, la "possibilité de tassement" est plus grande, mais par contre la contrainte verticale y est en général plus faible que si le milieu était homogène. Finalement, si l'hétérogénéité n'est pas trop importante " le risque de tassement " n'est sans doute pas très différent dans la couche réelle et dans le sol théorique homogène sur lequel a été fait le calcul.

Si par contre la couche rencontrée a des propriétés très différentes du reste du sol de fondation ce critère n'est plus réaliste. Alors, de deux choses l'une:

ou cette couche est très épaisse et il faut se servir des indications que peut fournir la géologie :

. ou cette couche est peu épaisse et constitue en quelque sorte une inclusion dans un ensemble relativement homogène: c'est ce cas que nous allons étudier maintenant.

\subsection{Critère en tassement}

Le risque de rencontrer une couche plus compressible que le sol environnant est très réel dans beaucoup de sites. Les renseignements fournis par la géologie permettent souvent de prévoir l'existence possible d'une telle couche mais ne permettent d'en prévoir ni la profondeur, ni l'épaisseur, ni l'étendue. Il est certain que les conséquences qu'une telle couche peut avoir sur le tassement de la fondation dépendent de son épaisseur, de sa compressibilité et de sa profondeur, paramètre qui va conditionner le choix de la profondeur de reconnaissance. Pour étudier ce problème, considérons une fondation rectangulaire de largeur $B$ et longueur $L$ reposant sur un sol contenant une telle couche.

Nous noterons (Fig. 14):

. H. la profondeur du toit de la couche très compressible :

- $\mathrm{H}_{2}$, l'épaisseur de cette couche :

. $E_{2}$, son module et $v$ son coefficient de Poisson :

, q, la surcharge apportée par la fondation, au niveau de sa base (cette surcharge se déduit de la pression moyenne, $p$, au niveau de la base de la fondation par la formule 16); nous supposons que cette surcharge $q$ est normale et uniformément distribuée.

Notre but est de déterminer l'accroissement, $\Delta \mathrm{w}$, du tassement de la surface du sol que provoquerait l'existence de la couche très compressible définie ci-dessus. Pour cela, nous écrivons :

$$
\Delta w=\Delta w_{2}-\Delta w_{1}
$$

avec :

$\Delta w_{2}$ : contribution apportée au tassement de la surface par la couche ci-dessus décrite :

$\Delta w_{1}$ : contribution qu'aurait apportée cette couche au tassement de la surface si elle avait eu les mêmes propriétés que le sol environnant.

Nous calculerons $\Delta w_{1}, \Delta w_{2}$ et, par conséquent, $\Delta w$ au coin de la fondation car c'est évidemment dans le cas d'une lentille de sol compressible située sous le coin que le risque de tassement différentiel, donc de désordres, est le plus grand. Pour calculer $\Delta w_{1}$ et $\Delta w_{2}$, il nous faut, au préalable, déterminer les contraintes verticales, $\sigma_{z}$, régnant dans la couche considérée. Pour faire cette détermination, nous supposerons que $\mathrm{H}_{2}$ est petit devant $\mathrm{H}$. Cette hypothèse est tout à fait acceptable dans le cas qui nous préoccupe car si $\mathrm{H}_{2}$ n'est pas petit devant $\mathrm{H}$, cela signifie, soit que la couche est proche de la surface (et dans ces conditions aucune profondeur de sondage, même déterminée à partir du critère en contrainte, ne permettra de l'ignorer). soit que située à une profondeur relativement importante son épaisseur est grande (dans ces conditions la géologie doit pouvoir signaler sa présence).

De cette hypothèse découlent deux conséquences importantes pour le calcul des contraintes verticales:

- la distribution de $\sigma_{z}$ est très peu perturbée par la présence de la couche mince et l'on pourra faire le calcul du tassement en utilisant la valeur de $\sigma_{\mathrm{z}}$ dans un sol homogène donnée par :

$$
\sigma_{\mathrm{Z}}=q \mathrm{O}_{\mathrm{Z}}\left(\frac{\mathrm{Z}}{\mathrm{B}^{\prime}}, \frac{\mathrm{L}}{\mathrm{B}}\right)
$$

(formule valable à la verticale du coin de la fondation)

la valeur de $\sigma_{z}$ est pratiquement constante sur toute la hauteur de la couche mince.

Connaissant, grâce aux remarques précédentes, la valeur de $\sigma_{z}$ dans la couche mince, on en déduit la contribution qu'elle apporte au tassement du coin de la fondation par la formule suivante

$$
\Delta w_{i}=q \frac{H_{2}}{E_{o e d i}} O_{z}\left(\frac{H}{B}, \frac{L}{B}\right)
$$

avec :

$$
\begin{aligned}
& i=1 \text { pour le calcul de } \Delta w_{1} \\
& i=2 \text { pour le calcul de } \Delta w_{2} \\
& E_{\text {oed }} \text { : module oedométrique. }
\end{aligned}
$$

Cette formule r'est autre que celle du tassement oedométrique. Son emploi se justifie car la répartition des contraintes verticales au niveau de la couche mince est telle qu'elle peut être considérée comme relativement uniforme sur une largeur assez grande vis-à-vis de l'épaisseur de cette couche : il est donc licite de négliger le déplacement latéral $\left(^{*}\right)$ et, donc, de faire un calcul "oedométrique ». De plus, la couche peut être une lentille de dimensions horizontales limitées, ce qui rapproche encore des conditions de l'oedomètre. Et, d'ailleurs, c'est dans le cas d'une lentille que le risque de tassement différentiel est le plus grand.

Les formules (8) et $(10)$ conduisent à :

$$
\begin{aligned}
\Delta w & =q H_{2}\left(\frac{1}{E_{\text {oed2 }}}-\frac{1}{E_{\text {oed } 1}}\right) O_{z^{\prime}}\left(\frac{H}{B}, \frac{L}{B}\right) \\
& =q \frac{H_{2}}{E_{\text {oed2 }}}\left(1-\frac{E_{\text {oed } 2}}{E_{\text {oed } 1}}\right) O_{z}\left(\frac{H}{B}, \frac{L}{B}\right)
\end{aligned}
$$

Négligeons $E_{\text {oed2 }} / E_{\text {oed1 }}$ vis-à-vis de 1, ce qui conduit à une légère surestimation de $\Delta w$. Par ailleurs, on connaît la formule classique :

$$
E_{\text {oed }}=E \frac{(1-v)}{(1+v)(1-2 v)}
$$

(*) Le tassement de la fondation est composé diune partie due au déplacement latéral du sol mais il s'agit essentiellement du déplacement latéral des couches voisines de la surface. 
(Nota : v étant le coefficient de Poisson du sol drainé, il ne peut pas avoir la valeur 0.5 qui annulerait le dénominateur).

D'où :

$$
\Delta w=q \frac{H_{2}}{E_{2}} \frac{1-v}{(1+v)(1-2 v)} \quad O_{z}\left(\frac{H}{B}, \frac{L}{B}\right)
$$

Posons ;

$$
\psi=\frac{\Delta w}{H_{2}} \frac{E_{2}}{q}=\frac{1-v}{(1+v)(1-2 v)} O_{z}\left(\frac{H}{B}, \frac{L}{B}\right)
$$

(Nota : l'expression analytique de $\mathrm{Oz}(\mathrm{H} / \mathrm{B}, \mathrm{L} / \mathrm{B})$ est connue d'après [15]).

Dans le cas d'un sol drainé, le coefficient de Poisson a, dans la plupart des cas, une valeur voisine de 0.3 . Nous avons donc retenu cette valeur pour établir le graphique de la figure 15 qui donne les valeurs numériques de $\Psi$ en fonction de $\mathrm{H} / \mathrm{B}$ et $\mathrm{L} / \mathrm{B}$. Inversement, si l'on se fixe une valeur admissible, $\Delta w_{\text {adm }}$, du tassement, la formule (14) ou la figure 15 permettent de déterminer la valeur de la profondeur $\mathrm{H}$ au-delà de laquelie la présence de la couche compressible hypothétique n'est pas dangereuse. En effet, au-delà de cette profondeur, la couche compressible n'augmenterait le tassement que d'une valeur inférieure à $\Delta \mathrm{w}_{\text {adm }}$. La profondeur $\mathrm{H}$ ainsi déterminée est donc la profondeur à laquelle il faut reconnaître le sol, en vertu du critère de tassement.

Pratiquement, on écrira $\Psi$ de la façon suivante :

$$
\psi\left(\frac{H_{r}}{B}\right)=\left(\frac{\Delta w}{B_{0}}\right)_{a d m} \frac{B_{0}}{H_{2}} \frac{E_{2}}{q}
$$

Fig. 15 Détermination de la profondeur de reconnaissance, $\mathrm{Hr}$, en fonction de $\psi$ qui est défini par la formule 15. Voir également la figure 5 pour la définition de certains termes

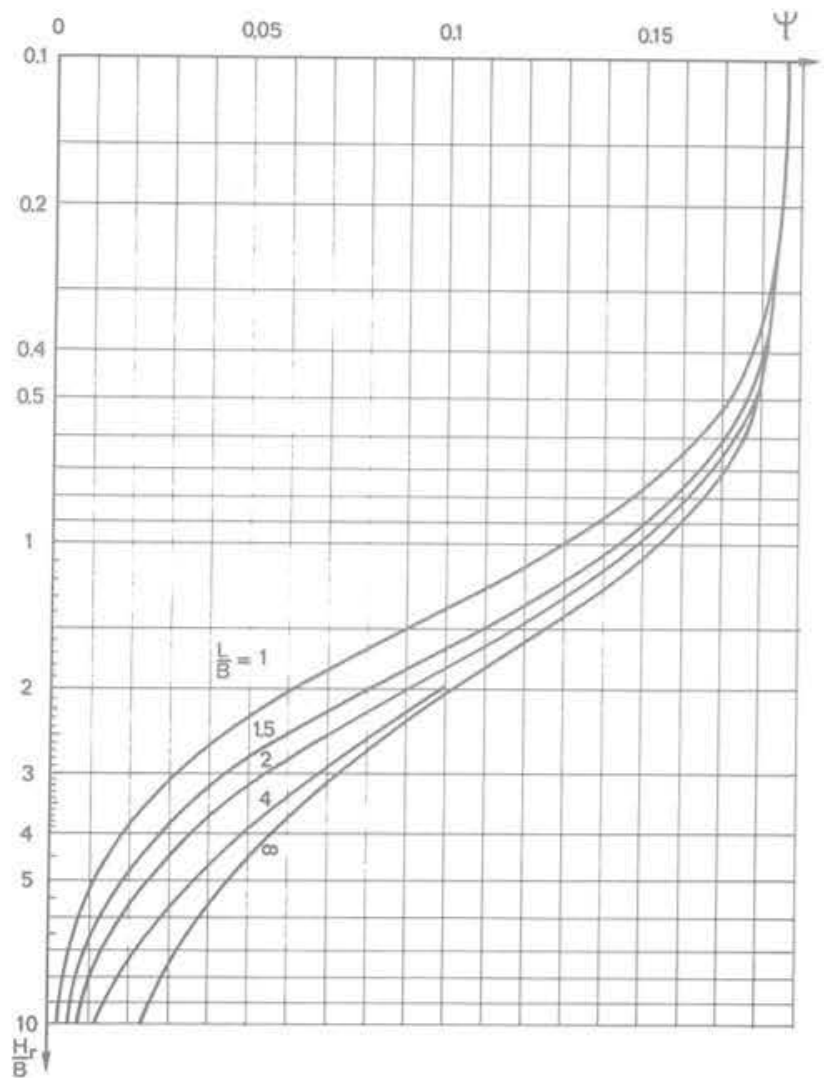

Connaissant les termes du membre de droite, on calcule $\Psi$ d'où l'on en déduit $\mathrm{Hr} / \mathrm{B}$ par simple lecture sur la figure 15 . Examinons en détail les termes de cette formule :

$H_{r} \quad$ est la profondeur (mesurée à partir de la base de la fondation) des sondages de reconnaissance qui doivent être réalisés (si l'on redoute la présence d'une lentille compressible) sous les quatre coins du bâtiment :

B est la largeur totale du bâtiment, que celui-ci soit fondé sur un radier ou sur des semelles (la figure 16 montre en effet qu'à partir d'une profondeur relativement faible la contrainte verticale ne dépend que très peu du type de fondation):

B. est la largeur de référence servant à estimer les effets du tassement différentiel (distance entre semelles, côté du radier,....);

$(\Delta w)$ est le tassement différentiel admissible ; autrement dit il s'agit de l'accroissement $\Delta w$ de tassement dû à la présence de la lentille compressible divisé par la largeur de référence Bo. L'ingénieur qui établit le projet de fondations peut le choisir en s'inspirant de recommandations de Skempton et Macdonald [19]: si $\Delta w / B_{0}>1 / 300$ on observe des désordres architecturaux et si $\triangle \mathrm{w} / \mathrm{B}_{0}>1 / 150$ on observe des désordres structuraux

$\mathrm{H}_{2} \quad$ est l'épaisseur supposée de la couche de module $\mathrm{E}_{2}$; à titre indicatif, il paraît raisonnable de prendre $\mathrm{H}_{2}$ entre 0.5 et 5 mètres :

Fig. 16 Isobares de la contrainte verticale $\sigma_{z}$ dans un milieu semi-infini ; à gauche dans le cas d'un radier infiniment long uniformément chargé, à droite dans le cas de quatre semelles parallèles [17]

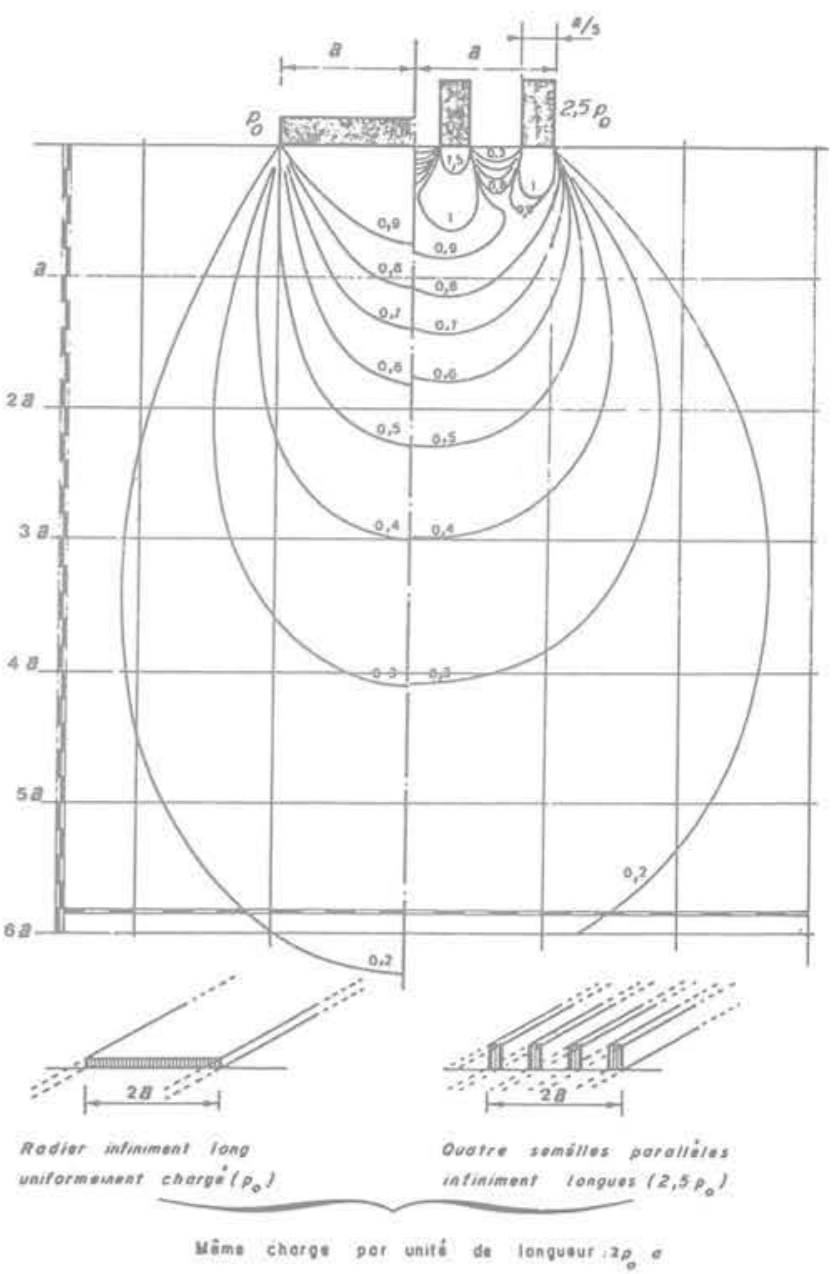


est la surcharge apportée par la fondation au niveau de sa base ; on démontre [17] qu'elle est donnée par la formule (fig. 17):

$$
q=p-\gamma_{0} D
$$

avec :

p : pression moyenne à la base de la fondation ( $p=$ charge totale y compris le poids de la fondation divisée par LB) :

$\gamma_{\mathrm{o}} \quad$ : poids volumique du sol situé entre la surface et le niveau de la base de la fondation:

D : profondeur de la base de la fondation.

\section{Applications pratiques et conclusions}

Dans cette dernière partie nous regroupons, à l'intention des lecteurs, des informations pratiques leur permettant d'utiliser rapidement les résultats présentés dans cette étude. Nous présentons également quelques exemples numériques puis des remarques en conclusion.

\subsection{Conseils pratiques pour la détermination de la} profondeur de reconnaissance

Trois critères ont été proposés pour la détermination de la profondeur de reconnaissance :

. un critère relatif à la stabilité (§ 1.2):

. deux critères relatifs au tassement : le «critère en contraintes " $(\S 2-1)$ et le " critère en tassement * (§ 2-2).

En principe, dans chaque cas, il est recommandé d'utiliser les trois critères et de retenir la plus grande des trois profondeurs ainsi obtenues.

Ces critères font intervenir deux types de paramètres. Il y a d'abord les paramètres qui définissent la fondation et qui sont communs aux trois critères (voir la figure 5):

. la longueur, $L$, et la largeur, B, du bâtiment ;

- la profondeur, D, prévue pour la base des fondations :

- la pression moyenne, p, à la base de la fondation (égale à la charge totale, y compris le poids de la fondation, divisée par la surface de la fondation d'où l'on tire la surcharge $q=p-\gamma_{0} D\left(\gamma_{0}\right.$ étant le poids volumique du sol au-dessus du niveau de la base de la fondation: on peut l'estimer forfaitairement de 18000 à $20000 \mathrm{~N} / \mathrm{m}^{3}$ selon que le sol est sec ou humide).

II $y$ a ensuite les paramètres propres à chacun des trois critères et qui, n'étant pas donnés a priori, impliquent une certaine décision de la part de l'ingénieur qui établit le projet de fondations. Examinons successivement les trois critères et, pour chacun d'eux, donnons la marche à suivre par l'ingénieur.

Pour le critère de stabilité, il faut connaître la cohésion, c. I'angle de frottement, $\varphi$, et le poids volumique, $\gamma$ (ce dernier pouvant être pris égal à $18000 \mathrm{~N} / \mathrm{m}^{3}$ pour un sol sec. $20000 \mathrm{~N} / \mathrm{m}^{3}$ pour un sol humide au-dessus de la nappe et $11000 \mathrm{~N} / \mathrm{m}^{3}$ au-dessous de la nappe) du sol qui sera au contact de la base de la fondation. L'ingénieur pourra estimer $c$ et $\varphi$ en faisant faire un trou à la pelle et en observant le terrain. A titre indicatif, voici quelques valeurs typiques:

- argile saturée (pour un calcul de pression portante immédiate) : $\varphi=0^{\circ}$ et $\mathrm{c}$ de l'ordre de $10^{4}$ ou $10^{5}$ $\mathrm{N} / \mathrm{m}^{2}$, selon la consistance ;

. argile saturée (pour un calcul de pression portante à long terme) : $\mathrm{c}=0, \varphi=15$ à $25^{\circ}$ :

, sols pulvérulents : $c=0$ et $\varphi=30^{\circ}$ (sable lâche), $35^{\circ}$ (sable compact) et $40^{\circ}$ (gravier).

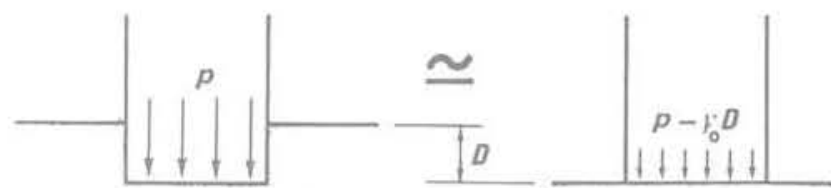

a

b

rig. 17 Relation entre la pression, $p$, réellement ap. pliquée par la fondation et la surcharge $q$ : celle-ci s'obtient en déduisant de $p$ la pression des terres enlevées [17]

Ce choix étant fait, l'ingénieur n'a plus qu’à utiliser le graphique correspondant:

. Fig. 10 pour $\varphi=0^{\circ}$

. Fig. 11 pour $\mathrm{c}=0$;

. Fig. 6 à 9 pour $c$ et $\varphi$ différents de 0 .

Examinons maintenant les deux critères relatifs au risque de tassement: le critère en contraintes et le critère en tassement.

Pour le critère en contraintes, il suffit de connaître le poids volumique, $\gamma$, du sol qui sera en contact avec la fondation lon peut prendre $18000 \mathrm{~N} / \mathrm{m}^{3}$ pour le sol sec. $20000 \mathrm{~N} / \mathrm{m}^{3}$ pour le sol humide au-dessus de la nappe et $11000 \mathrm{~N} / \mathrm{m}^{3}$ pour le sol au-dessous de la nappe) et il faut choisir la valeur du paramètre $\mathbf{m}$, défini par (6), selon le risque accepté. On pourra s'inspirer de (7) pour faire ce choix. Ensuite il suffit d'utiliser le graphique de la figure 13.

Pour le critère en tassement, il faut faire deux choix : - il faut choisir la valeur du tassement admissible, $\left(\Delta \mathrm{W} / \mathrm{B}_{\mathrm{o}}\right)_{\text {adm }}$; pour cela, on pourra s'inspirer des recommandations de Skempton et Macdonald indiquées plus haut, après la formule (15) ;

. il faut choisir l'épaisseur, $\mathrm{H}_{2}$, et le module de déformation, $E_{2}$, de la couche compressible dont on redoute la présence; pour le choix de $\mathrm{H}_{2}$ on pourra s'inspirer d'éventuels renseignements fournis par la géologie (une valeur de 0.5 à 5 mètres parait raisonnable): pour $E_{2}$ il faudra prendre une valeur nettement inférieure à celle du module, E, du sol qui sera en contact avec la fondation (par exemple, $E_{2} / E$ de l'ordre de $1 / 10$ '.

Ces choix étant faits, on calcule $\Psi$ par la formule (15) et I'on en déduit une valeur de la profondeur de reconnaissance par la figure 15.

Pour fixer les idées, faisons une application numérique. Considérons trois bâtiments :

- une tour de base carrée, $15 \times 15 \mathrm{~m}$, de 20 étages exerçant sur le sol une surcharge de $200000 \mathrm{~N} / \mathrm{m}^{2}$; , un immeuble de base rectangulaire, $20 \times 80 \mathrm{~m}$, de 6 étages, exerçant sur le sol une surcharge de $60000 \mathrm{~N} / \mathrm{m}^{2}$

- un marché de $50 \mathrm{~m}$ de long par $20 \mathrm{~m}$ de large dont la superstructure repose sur des poteaux chargés à 400000 Newtons et formant une maille carrée de $10 \mathrm{~m}$ de côté (soit 18 poteaux pour une charge totale de 7200000 Newtons).

Le sol, tel qu'il apparait au voisinage de la surface, est un silt non saturé (la nappe est très profonde), assez compact. On peut lui attribuer les propriétés suivantes :

$\varphi=25^{\circ}$

$\gamma=20000 \mathrm{~N} / \mathrm{m}^{3}$

$\mathrm{c}=40000 \mathrm{~N} / \mathrm{m}^{2}$

$E=5$ a $1010^{6} \mathrm{~N} / \mathrm{m}^{2}$ 


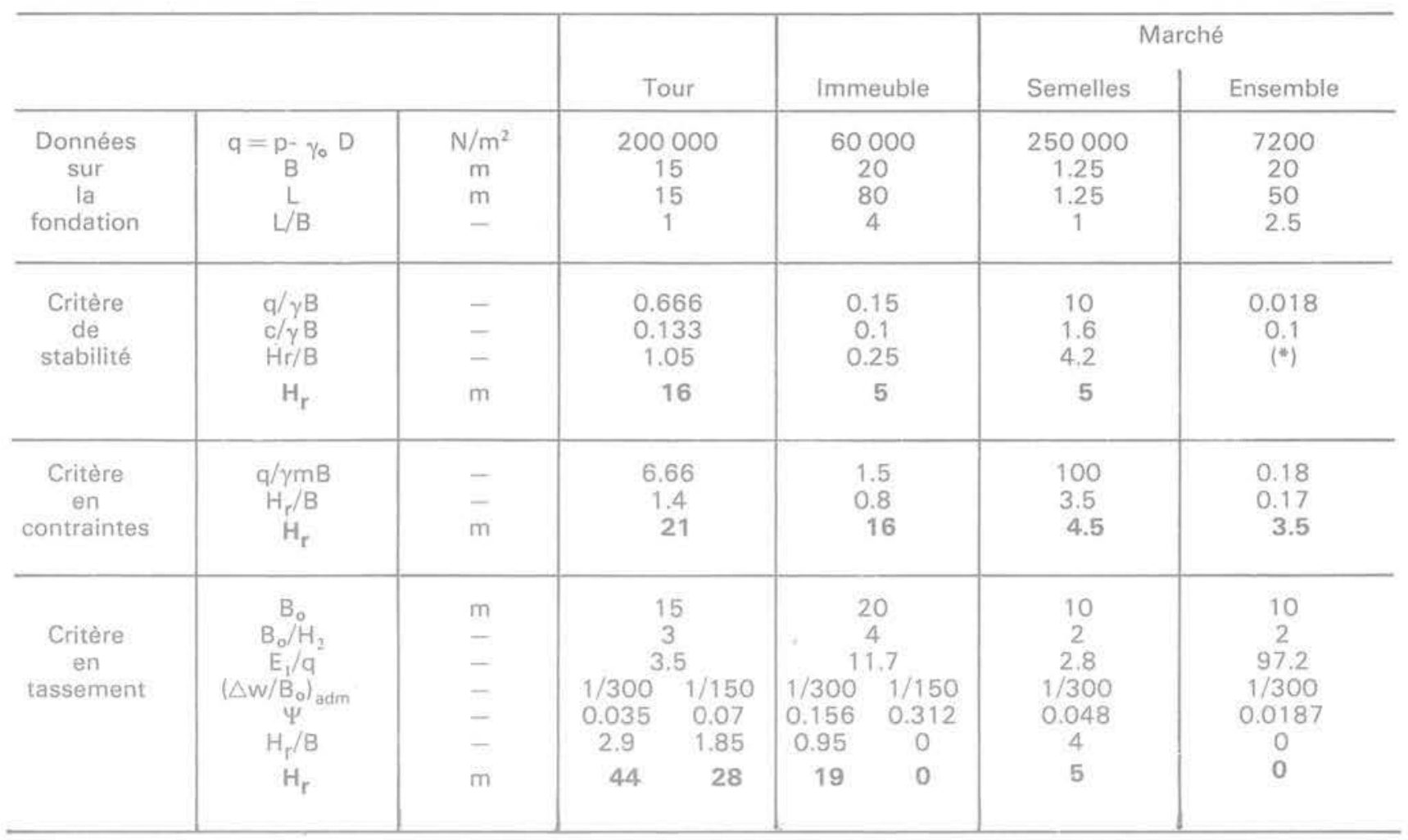

Tableau 2 Détail des valeurs de l'exemple numérique. (") Calcul impossible car les valeurs sortent du graphique

Le détail des calculs et les résultats se trouvent dans le Tableau 2. Il n'y a pas de difficulté particulière. Signalons cependant les quelques points suivants :

. pour le critère en stabilité il a fallu interpoler entre les figures 7 et 8 ;

. pour le critère en contraintes, nous avons choisi $m=0.1$, ce qui est plutôt prudent

, pour le critère en tassement nous avons pris $\mathrm{H}_{2}=5 \mathrm{~m}$ (ce qui est plutôt grand) et $E_{2}=$ $700000 \mathrm{~N} / \mathrm{m}^{2}$ (ce qui est de l'ordre de $\mathrm{E} / 10$ )

. pour le marché, nous avons fait deux calculs, l'un en considérant le marché dans son ensemble $(B=20 \mathrm{~m}$ et $\mathrm{L}=50 \mathrm{~m})$, lautre en considérant chaque semelle isolément (nous avons alors pris, compte tenu des caractéristiques du sol, $q=250000 \mathrm{~N} / \mathrm{m}^{2}$ d'où $\mathrm{L}=\mathrm{B}=1.25 \mathrm{~m}$ ).

On remarquera que, notamment pour la tour, le critère en stabilité conduit à une profondeur plus faible que le critère en tassement. Ce résultat est satisfaisant car il signifie que si la profondeur choisie pour la reconnaissance était faible. on aurait plutôt un risque de tassement que de rupture.

En comparant tous les résultats, il semble logique de retenir :

$.5 \mathrm{~m}$ pour le marché :

. 16 \19 m pour l'immeuble selon le degré de tolèrance sur le tassement différentiel :

. 28 à $44 \mathrm{~m}$ pour la tour, selon le degré de tolérance sur le tassement différentiel.

Rappelons que ces profondeurs de reconnaissance sont mesurées à partir de la base de la fondation : il faut leur rajouter la valeur de D. Enfin, il est toujours recommandé. pour l'un au moins des sondages, d'adopter une profondeur plus grande par souci de sécurité.

\subsection{Conclusions}

Pour conclure, il semble intéressant de comparer les critères que nous proposons dans cette étude à ce que font habituellement les ingénieurs. En fait, dans les traités de Mécanique des Sols, on ne donne pratiquement jamais aux ingénieurs de règles pour le choix de la profondeur des reconnaissances, hormis quelques valeurs en fonction de la largeur de la fondation. Ces règles sont évidemment insuffisantes car elles ne font pas intervenir des paramètres qui, d'après notre étude, sont importants, comme :

$$
\begin{aligned}
& \text {. la longueur de la fondation : } \\
& \text {, la surcharge apportée par la fondation sur le sol : } \\
& \text {, les propriétés du sol: } \\
& \text {, le risque de tassement admissible. }
\end{aligned}
$$

Ceci montre le parti que les ingénieurs peuvent tirer de nos critères pour améliorer sécurité et économie. En particulier, l'ingénieur qui doit faire un plan de reconnaissance du sol pour un ensemble de bâtiments très variés sur un terrain donné pourra, grâce à nos critères, répartir judicieusement le coût de sa campagne de reconnaissance.

Bien entendu, on pourra regretter la lourdeur de la méthode qui impose lutilisation de plusieurs graphiques. C'est à ce prix qu'il faut payer la prise en compte des nombreux paramètres qui conditionnent la qualité du résultat. Les indications pratiques du $\S 3-1$ doivent notablement faciliter le travail de l'ingénieur. Cependant (et c'est la conclusion classique) l'ingénieur doit faire appel à son expérience et à son jugement pour faire judicieusement les choix des valeurs des paramètres sans lesquels les méthodes proposées ne donneraient pas de résultats satisfaisants. 


\section{Références Bibliographiques}

[1] P. HABIB et L. SUKLJE-Etude de la stabilité des fondations sur une couche d'argile d'épaisseur limitée. An. I.T.B.T.P. $n^{\circ} 83$, S.F. 15 , (Nov. 1954).

[2] Y. TCHENG - Fondations superficielles en milieu stratifié. Proc. 4th Inf. Conf. S.M. (London, 1957).

[3] J. HAERINGER - Contribution à l'étude de la force portante des fondations de surface en milieu pulvérulent ả deux dimensions. Thèse (Grenoble, 1964).

[4] J.D. BROWN et G. MEYERHOF - Experimental study of bearing capacity on layered clays. Proc. of Int. Conf. S.M. 3 (Mexico, 1969).

[5] A.S. KANANYAN - Experimental investigation of the stability of foundation beds of finite thickness. Soil Mech. and Found. Eng., 5, (Sept.-Oct. 1971).

[6] Y. LEBEGUE - Contrainte à I'interface d'un milieu constitué par du sable surmontant de l'argile molle. Communication aux Journées Françaises de Mécanique des Sols (Mai 1971). Bulletin de liaison des LPC (Juin 1972), 160.

[7] J.P. GIROUD, TRAN-VO-NHIEM et J.P. OBIN - Tables pour le calcul des fondations. Volume 3 : Force portante, Dunod (Paris 1973).

[8] J. MANDEL et J. SALENCON - Force portante d'un sol sur assise rigide. Proc, 7 th Int. Conf. S.M., 3 (Mexico, 1969).

[9] J. MANDEL et J. SALENCON - Force portante d'un sol sur assise rigide (Etude théorique). Geotechnique (1972).
[10] Y. KOIZUMI - Bearing capacity of footing resting on two-layers (sand-clay). Report of Architectural Institute of Japan (1959) (en Japonais).

[11] $\mathrm{H}$. YAMAGUCHI - Practical formula of gearing value for two-layered ground. Proc. 2nd Asian Reg. Conf. S.M. (1963), 176.

[12] S.J. BUTTON - The bearing capacity of footings resting on two-layers cohesive subsoil. Proc. 3rd Int. Conf. S.M. (Zurich, 1953).

[13] J.P. OBIN - Force portante en déformation plane d'un sol verticalement non-homogène. Thèse de doctorat de spécialité. Université Scientifique et Médicale de Grenoble (février 1972).

[14] D.M. BURMISTER - Stress and displacement characteristics of a two layer rigid box soil system: influence diagrams and practical applications. Proc. HRB 35 (January 1956), 773-814.

[15] J.P. GIROUD - Tables pour le calcul des fondations. Tome 2, Dunod (1972).

[16] J.P. GIROUD - Guide for depth of foundation exploration. Discussion Journal of the Soil Mechanics and Foundation Division ASCE. No SM 6, Proc. Pap. 7643 (November 1970), 2140-2143.

[17] J.P.GIROUD - Le tassement des fondations superficielles PUG (Grenoble, 1975).

[18] Normes soviétiques pour le calcul des fondations des bátiments et ouvrages. SNIP, Strojezdat, (Moscou, 1975).

[19] A.W. SKEMPTON and D.H. MACDONALD - The allowable settiement of buildings. Proc. ICE, 5,3 (December 1956), 727-784. 
